\title{
THE SPECTRAL ENERGY DISTRIBUTION AND EMISSION-LINE PROPERTIES OF THE NARROW-LINE SEYFERT 1 GALAXY ARAKELIAN 564
}

\author{
P. Romano, ${ }^{1,2}$ S. Mathur, ${ }^{1}$ T. J. Turner ${ }^{3,4}$ S. B. Kraemer,${ }^{5}$ D. M. Crenshaw, ${ }^{6}$ B. M. Peterson, ${ }^{1}$ R. W. Pogge ${ }^{1}$ W. N. Brandt ${ }^{7}$ \\ I. M. George, ${ }^{3,4}$ K. Horne, ${ }^{8}$ G. A. Kriss, ${ }^{9}$ H. Netzer, ${ }^{10}$ O. Shemmer, ${ }^{10}$ and W. Wamsteker ${ }^{11}$ \\ Received 2003 August 4; accepted 2003 November 7
}

\begin{abstract}
We present the intrinsic spectral energy distribution (SED) of the narrow-line Seyfert 1 galaxy (NLS1) Ark 564, constructed with contemporaneous data obtained during a multiwavelength, multisatellite observing campaign in 2000 and 2001. We compare this SED with that of the NLS1 Ton S180 and with those obtained for broad-line Seyfert 1 galaxies to infer how the relative accretion rates vary among the Seyfert 1 population. Although the peak of the SED is not well constrained, in our parameterization most of the energy of this object is emitted in the 10-100 eV regime, constituting roughly half of the emitted energy in the optical/X-ray ranges. This is consistent with a primary spectral component peaking in the extreme-UV/soft X-ray band and with diskcorona models, hence high accretion rates. Indeed, we estimate that $\dot{m} \approx 1$. We also address the issue of the energy budget in this source by examining the emission lines observed in its spectrum, and we constrain the physical properties of the line-emitting gas through photoionization modeling. The available data suggest that the line-emitting gas is characterized by $\log n \approx 11$ and $\log U \approx 0$ and is stratified around $\log U \approx 0$. Our estimate of the radius of the $\mathrm{H} \beta$-emitting region $R_{\mathrm{BLR}}^{\mathrm{H} \beta} \approx 10 \pm 2$ 1t-days is consistent with the $R_{\mathrm{BLR}}^{\mathrm{H} \beta}$-luminosity relationships found for Seyfert 1 galaxies, which indicates that the narrowness of the emission lines in this NLS1 is not due to the broad-line region being relatively farther away from the central mass than in broad-line Seyfert 1 galaxies of comparable luminosity. We also find evidence for supersolar metallicity in this NLS1. We show that the emission lines are not good diagnostics for the underlying SEDs and that the absorption line studies offer a far more powerful tool to determine the ionizing continuum of active galactic nuclei, especially if one is comparing the lower and higher ionization lines.
\end{abstract}

Subject headings: galaxies: active — galaxies: individual (Arakelian 564) — galaxies: nuclei — galaxies: Seyfert

On-line material: color figure

\section{INTRODUCTION}

The population of Seyfert 1 galaxies has a widely used subclassification into narrow-line Seyfert 1 galaxies (NLS1s) and broad-line Seyfert 1 galaxies (BLS1s). While this classification appears to make an arbitrary distinction based on the widths of the optical emission lines [NLS1s having $\operatorname{FWHM}(\mathrm{H} \beta) \lesssim 2000 \mathrm{~km} \mathrm{~s}^{-1}$; Goodrich 1989], this is in fact

\footnotetext{
${ }^{1}$ Department of Astronomy, Ohio State University, 140 West 18th Avenue, Columbus, $\mathrm{OH} 43210$.

2 Current address: INAF-Osservatorio Astronomico di Brera, Via E. Bianchi 46, 23807 Merate (LC), Italy; romano@merate.mi.astro.it.

${ }^{3}$ Laboratory for High Energy Astrophysics, NASA Goddard Space Flight Center, Code 660, Greenbelt, MD 20771.

4 Joint Center for Astrophysics, Physics Department, University of Maryland, Baltimore County, 1000 Hilltop Circle, Baltimore, MD 21250.

${ }^{5}$ Catholic University of America and Laboratory for Astronomy and Solar Physics, NASA Goddard Space Flight Center, Code 681, Greenbelt, MD 20771.

${ }^{6}$ Department of Physics and Astronomy, Georgia State University, Astronomy Offices, 1 Park Place South SE, Suite 700, Atlanta, GA 30303.

${ }^{7}$ Department of Astronomy and Astrophysics, Pennsylvania State University, 525 Davey Laboratory, University Park, PA 16802.

${ }^{8}$ School of Physics and Astronomy, University of St. Andrews, St. Andrews KY16 9SS, UK

9 Space Telescope Science Institute, 3700 San Martin Drive, Baltimore, MD 21218.

${ }^{10}$ School of Physics and Astronomy and the Wise Observatory, Raymond and Beverly Sackler Faculty of Exact Sciences, Tel Aviv University, Tel Aviv 69978 , Israel.

11 European Space Agency, P.O. Box 50727, 28080 Madrid, Spain.
}

an extremely useful scheme since the X-ray properties of the two subclasses are systematically different. As a class, NLS1s show rapid and large-amplitude X-ray variability (Boller, Brandt, \& Fink 1996; Turner et al. 1999b), with the excess variance (Nandra et al. 1997a) typically an order of magnitude larger than that observed for samples of BLS1s with the same luminosity distribution (Turner et al. 1999b; Leighly 1999a). Analogously, the spectral properties also vary across the Seyfert population, with NLS1s showing systematically steeper spectra than those of BLS1s in both the soft and hard X-ray bands (Boller et al. 1996; Brandt, Mathur, \& Elvis 1997; Turner, George, \& Nandra 1998; Leighly 1999b; Vaughan et al. 1999).

One increasingly popular hypothesis to explain the differences in X-ray properties across the Seyfert population is that NLS1s have relatively low masses for the central black hole compared with BLS1s with similar luminosities. Smaller black hole masses naturally explain both the narrowness of the optical emission lines, which are generated in gas that has relatively small Keplerian velocities, and the extreme X-ray variability, since the primary emission would originate in a smaller region around the central engine (e.g., Laor et al. 1997). Given that NLS1s have comparable luminosity to that of the BLS1s, Pounds, Done, \& Osborne (1995) suggested that they must be emitting at higher fractions of their Eddington luminosity; hence, higher fractional accretion rates ( $\dot{m}=\dot{M} / \dot{M}_{\text {Edd }}$ ) are also required. The closer the luminosity is to the Eddington limit (and the lower the black hole mass), the 
greater the fraction of the energy emitted by the accretion disk in the soft X-rays (Ross, Fabian, \& Mineshige 1992). Thus, NLS1s might be expected to show disk components that peak at higher energies than for BLS1s. Pounds et al. (1995) and Maraschi \& Haardt (1997) noted that soft photons from the disk may Compton-cool hard X-rays from the corona and cause the observed steep spectra.

Given the dependence of the peak energy of the disk emission on the accretion rate, the spectral energy distribution (SED) of an active galactic nucleus (AGN) will provide critical information about accretion rates and conditions close to the disk. In particular, examination of the SED of an NLS1 and comparison with that obtained for BLS1s will offer insight into the relative accretion rates across the Seyfert population. However, measuring the SED requires observations taken simultaneously over a long wavelength baseline stretching from infrared to hard X-ray wavelengths, and the determination of the UV/X-ray continuum in AGNs is still difficult because of the severe attenuation by even small amounts of Galactic interstellar gas along the line of sight. Another complication comes from intrinsic reddening, i.e., reddening associated with the active nucleus itself. Indeed, the steeper UV/blue continua observed in NLS1s (when compared with AGN spectrum composites) can be attributed at least in part to reddening, although the ionization of the absorbing material and its location with respect to the accretion source is still not well determined (Constantin \& Shields 2003).

Arakelian 564 (Ark 564, IRAS 22403+2927, MGC +05-53012 ) is a bright, nearby NLS1 galaxy, with $z=0.02467$ and $V=14.6$ mag (de Vaucouleurs et al. 1991) and a mean 2$10 \mathrm{keV}$ luminosity $L_{2}-10 \mathrm{keV} \approx 2.4 \times 10^{43} \mathrm{ergs} \mathrm{s}^{-1}$ with flux variations of a factor of a few in a few thousand seconds (Turner et al. 2001, hereafter Paper I). It was the object of an intense multiwavelength monitoring campaign that included simultaneous observations from $A S C A$ (2000 June 1 to July 6; Paper I; Pounds et al. 2001; Edelson et al. 2002), XMMNewton (2000 June 17; Vignali et al. 2004), Chandra (2000 June 17; Matsumoto, Leighly, \& Marshall 2002), Hubble Space Telescope (HST; 2000 May 9-July 8; Collier et al. 2001, hereafter Paper II; Crenshaw et al. 2002, hereafter Paper IV), FUSE (2001 June 29-30; Romano et al. 2002, hereafter Paper V), and many ground-based observatories as part of an AGN Watch ${ }^{12}$ project (1998 November-2001 January; Shemmer et al. 2001, hereafter Paper III). Ark 564 has shown a strong associated UV absorber (Crenshaw et al. 1999; Paper II; Paper IV; Paper V). There are indications that it also possesses a warm X-ray absorber, as seen by the narrow absorption lines of $\mathrm{O}$ VII and $\mathrm{O}$ VIII detected in a Chandra spectrum (Matsumoto et al. 2002), and that the UV and X-ray absorbers in Ark 564 are physically related, possibly identical, and may be spatially extended along the line of sight (Paper V).

In this paper we present a contemporaneous SED of Ark 564, based on the extensive monitoring of 2000. In $\S 2$ we describe the observations and data reduction, summarize the main results of the monitoring campaign, and describe the adopted method for reddening correction. In $\S 3$ we present the SED of Ark 564. In $\S 4$ we constrain the mean physical properties of the line-emitting gas through photoionization modeling. In $\S 5$ we discuss some implications of our investigation. Finally, our results are summarized in $\S 6$.

\section{OBSERVATIONS}

\subsection{Data Reduction}

Table 1 summarizes the log of the observations of Ark 564 used in this study. Columns (1) and (2) report the observatory and instrument that obtained the data; column (3) shows the date on which the data were obtained; column (4) lists the ranges of energy and wavelength we used for our work; column (5) lists the total exposure times and the slit size, along with other relevant notes for a particular observation; and column (6) reports the references to the papers in which the data were first published. Most of the data have been published already, with the exception of the $X M M$ data, which will be published in Vignali et al. (2004); therefore, here we only briefly summarize their reduction and analysis, referring the reader to the original papers for further details and to $\S 2.2$ for a summary of the main results.

${ }^{12}$ All publicly available data and complete references to published AGN
Watch papers can be found at http://www.astronomy.ohio-state.edu/ agnwatch.

TABLE 1

OBSERVING LOG FOR ARK 564

\begin{tabular}{|c|c|c|c|c|c|}
\hline $\begin{array}{l}\text { Observatory } \\
\text { (1) }\end{array}$ & $\begin{array}{l}\text { Instrument } \\
\text { (2) }\end{array}$ & $\begin{array}{l}\text { UT Dates } \\
\text { (3) }\end{array}$ & $\begin{array}{c}\text { Wavelength/Energy }{ }^{\mathrm{a}} \\
\text { (4) }\end{array}$ & $\begin{array}{l}\text { Notes } \\
(5)\end{array}$ & $\begin{array}{l}\text { References } \\
\text { (6) }\end{array}$ \\
\hline ASCA & & 2000 Jun $1-$ Jul 6 & $0.75-9.76 \mathrm{keV}$ & $2.98 \mathrm{Ms}$, continuous ${ }^{\mathrm{b}}$ & 1 \\
\hline XMM-Newton ............. & & 2000 Jun 17 & $0.3-8 \mathrm{keV}$ & & 2 \\
\hline 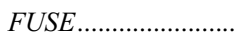 & & 2001 Jun 29-30 & $1000-1175 \AA$ & $63 \mathrm{ks} ; 30^{\prime \prime} \times 30^{\prime \prime}(\mathrm{LWRS})$ & 3 \\
\hline 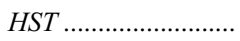 & STIS/G140L & 2000 May 9-Jul 8 & $1175-1711 \AA$ & $554304 \mathrm{~s} ; 52^{\prime \prime} \times 0^{\prime \prime} .5$ & 4,5 \\
\hline$H S T$......................... & STIS/G230L & 2000 May 9-Jul 8 & $1711-3143 \AA$ & $24216 \mathrm{~s} ; 52^{\prime \prime} \times 0.5$ & 4,5 \\
\hline 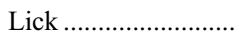 & & 1980 & $3170-4160 \AA$ & & 6 \\
\hline 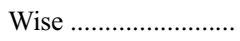 & FOSC & 1998 Nov-2001 Jan & $4160-7790 \AA$ & & 7 \\
\hline 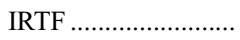 & SPEX & 2000 Oct 11,13 & $8200-24000 \AA^{\mathrm{c}}$ & $\sim 30$ minutes; $15^{\prime \prime} \times 00^{\prime \prime} 8$ & 8 \\
\hline 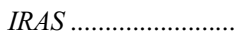 & & $\ldots$ & $12,25,60,100 \mu \mathrm{m}$ & & 9 \\
\hline
\end{tabular}

a Observed-frame wavelength/energy bands utilized.

${ }^{\mathrm{b}}$ Except for gaps due to Earth occultation and passage of the spacecraft through the South Atlantic Anomaly.

${ }^{\mathrm{c}}$ Only a few continuum points were used for this work.

REFERENCES.-(1) Turner et al. 2001; (2) Vignali et al. 2004; (3) Romano et al. 2002; (4) Collier et al. 2001; (5) Crenshaw et al. 2002; (6) D. E. Osterbrock 2002, private communication; (7) Shemmer et al. 2001; (8) Rodríguez-Ardila et al. 2002b and references therein; (9) Moshir et al. 1990. 
The ASCA data (Paper I) were obtained starting from 2000 June 1 (Julian Date 2,451,697.024), for a total exposure time of $\sim 2.98 \mathrm{Ms}$, and were reduced using standard techniques as described in Nandra et al. (1997a) with the methods and screening criteria utilized by the Tartarus ${ }^{13}$ database (Turner et al. 1999b). Data screening yielded an effective exposure time of $\sim 1.11 \mathrm{Ms}$ for the SISs and $\sim 1.29 \mathrm{Ms}$ for the GISs. The degradation of the low-energy response of the SIS detectors was corrected for with the method of Yaqoob et al. $(2000)^{14}$; i.e., we parameterized the efficiency loss with a time-dependent absorption $\left[N_{\mathrm{H}}(\mathrm{SIS} 0)=7.5 \times 10^{20} \mathrm{~cm}^{-2}, N_{\mathrm{H}}(\mathrm{SIS} 1)=1.05 \times\right.$ $10^{21} \mathrm{~cm}^{-2}$; Paper I].

FUSE observed Ark 564 for 63 ks starting from 2001 June 29 (Paper V). The observations consisted of 24 exposures performed in photon address (time-tag) mode through the $30^{\prime \prime} \times 30^{\prime \prime}$ low-resolution (LWRS) aperture. The data were reduced with CalFUSE (ver. 2.0.5) ${ }^{15}$ as a single continuous exposure. As a result of a high-voltage anomaly and data screening, the effective on-source times were $41 \mathrm{ks}$ in detector $1 \mathrm{~A}, 39 \mathrm{ks}$ in detector $1 \mathrm{~B}, 58 \mathrm{ks}$ in detector $2 \mathrm{~A}$, and $62 \mathrm{ks}$ in detector $2 \mathrm{~B}$. We discarded from further analysis the $\mathrm{SiC} 1 \mathrm{~A}$ and $\mathrm{SiC} 1 \mathrm{~B}$ spectra because of a high-voltage anomaly and the LiF1B spectrum since it showed wavelength-dependent differences in flux of up to $30 \%-50 \%$ compared with the LiF1A. We rebinned the full spectrum in a linear wavelength scale using $0.6 \AA$ bins (100 pixels, effective resolution of $20 \mathrm{~km}$ $\mathrm{s}^{-1}$ ). Comparison with the flux level of the HST spectrum indicates that in 2001 June, when the FUSE spectrum was obtained, the source was $\sim 1.3$ times brighter than in 2000 May-July, when the HST spectrum was obtained. We therefore scaled the FUSE fluxes by 0.75 to match the HST data. As noted in Paper $\mathrm{V}$, this is not inconsistent with a combination of effects such as flux intercalibration uncertainties and, most importantly, source flux variability.

The HST data (Papers II and IV) were obtained with the Space Telescope Imaging Spectrograph (STIS) in 46 visits between 2000 May 9 and July 8 (the first five visits were separated by intervals of 5 days, the remaining by 1 day). The spectra were obtained through the $52^{\prime \prime} \times 0.0^{\prime \prime} 5$ slit and the lowresolution G140L and G230L gratings, which yield a spectral resolution of $\sim 1.2$ and $3.2 \AA$ in the $1150-1730$ and $1570-$ $3150 \AA$ ranges, respectively. The data were reduced using the IDL software developed at NASA's Goddard Space Flight Center for the STIS Instrument Definition Team (Lindler 1998). The spectra have been corrected for small wavelength intercalibration uncertainties following Korista et al. (1995). The uncertainty in the relative wavelength calibration is on the order of 0.6 and $1.7 \AA$ for the G140L and G230L gratings, respectively. A separate mean spectrum was created for the G140L and G230L gratings separately, given the different resolutions.

In the optical we combined two spectra. The first one, which covers the 3170-4160 $\AA$ wavelength range with a mean spectral resolution of $0.62 \AA$, was obtained in 1980 at Lick Observatory (D. E. Osterbrock 2002, private communication). The second one is the mean of the spectra taken between 1998 November and 2001 January at the Tel Aviv University Wise Observatory (Paper III; resolution of $\sim 10 \AA$ ). The host galaxy starlight contribution has been estimated by measuring its

\footnotetext{
13 See http://tartarus.gsfc.nasa.gov.

14 See http://lheawww.gsfc.nasa.gov/ yaqoob/ccd/nhparam.html.

15 See http://fuse.pha.jhu.edu/analysis/calfuse.html.
}

flux through point-spread function (PSF) fitting to field stars in $V$-band images of the galaxy taken at Wise Observatory, which corresponds to $\sim 40 \%$ of the total light at $5200 \AA$, i.e., $F_{\text {gal }}=$ $2.4 \times 10^{-15} \operatorname{ergs~s}^{-1} \mathrm{~cm}^{-2} \AA^{-1}$ (Paper III). Given the limited resolution (dominated by a seeing disk of $\sim 2$ ". 5 ), it was not possible to separate the components of the host galaxy (bulge and bar) from PSF; hence, we subtracted from the mean spectrum a constant host contribution of $F_{\text {gal }}$. No scaling was necessary between the two spectra, in agreement with the lowamplitude variations of the continuum found in Paper II ( $\sim 6 \%$ over a month long observation) and Paper III $(\sim 10 \%$ during a 2 yr monitoring). Indeed, there is growing evidence that while NLS1s show very rapid and giant X-ray variability, they show only slow and minor optical variability (O. Shemmer et al. 2003, in preparation).

To extend the SED in the IR, we derived four continuum points between $\sim 10,000$ and 24,000 $\AA$ from Rodríguez-Ardila et al. (2002a, 2002b), which were obtained on 2000 October 11 and 13 with the NASA $3 \mathrm{~m}$ Infrared Telescope Facility (IRTF) and the SPEX spectrometer. We also retrieved archival IRAS flux measurements at 12, 25, 60, and $100 \mu \mathrm{m}$ (Moshir et al. 1990) through the NASA/IPAC Extragalactic Database (NED).

Figure 1 shows the contemporaneous SED of Ark 564 before correction for intervening (and intrinsic; see $\S 2.3$ ) absorption is applied. We note that while the HST and Wise spectra are simultaneous (as well as simultaneous with the $A S C A$ spectrum), the FUSE and Lick spectra were obtained $1 \mathrm{yr}$ later and $20 \mathrm{yr}$ earlier, respectively. The far-UV (FUV)/optical rest-frame spectrum of Ark 564 covering the 1000-7790 $\AA$ wavelength region is presented in Figure 2, spectrum $a$.

\subsection{Summary of Results from the Multiwaveband Observations}

The continuum fit to the mean ASCA spectrum (with a power-law model modified by Galactic absorption, $N_{\mathrm{H}}=$ $6.4 \times 10^{20} \mathrm{~cm}^{-2}$; Dickey \& Lockman 1990) yields a slope $\Gamma=2.538 \pm 0.005$ (Paper I). The strong excess of emission

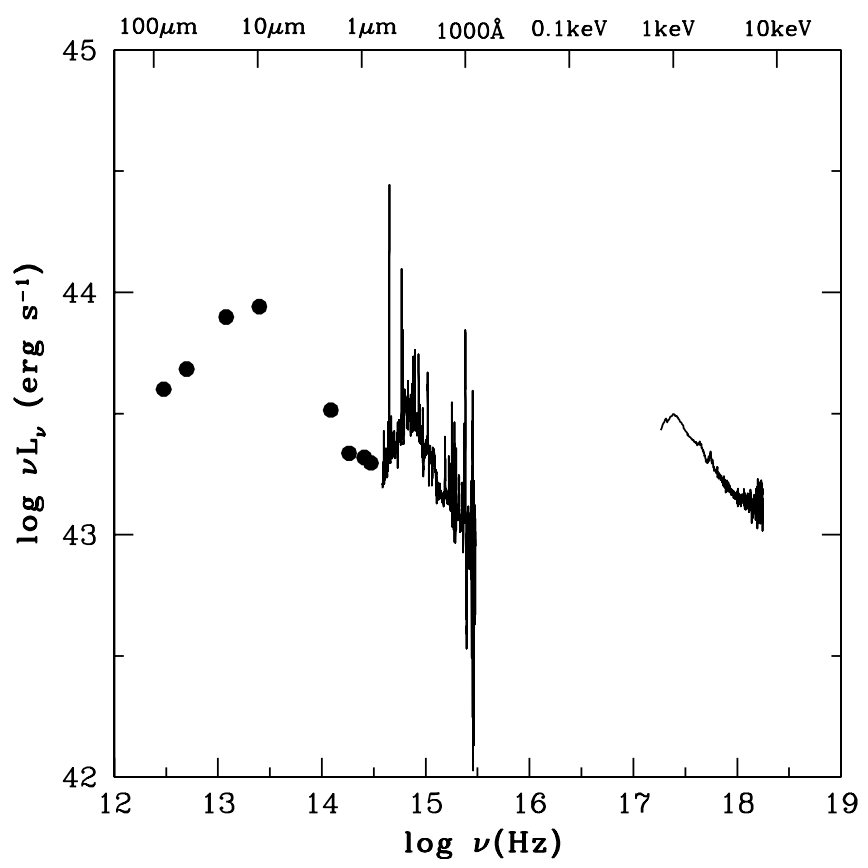

FIG. 1.-Quasi-simultaneous SED of Ark 564 (§ 2.1). The data have not been corrected for reddening and are in the observed frame. 


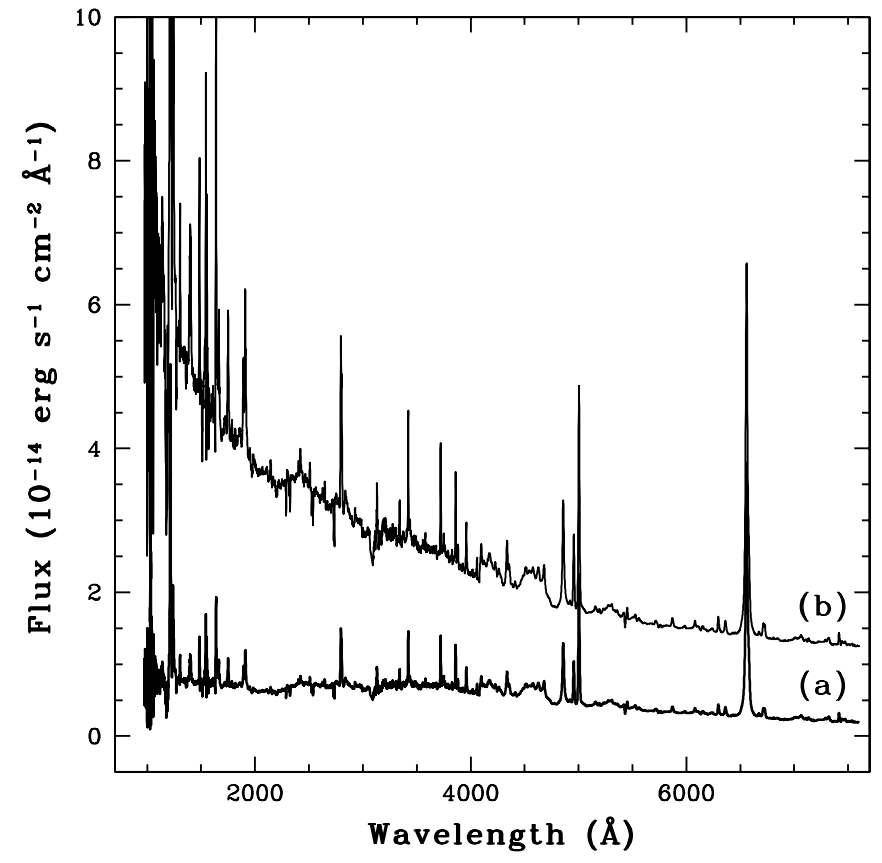

FIG. 2.-(a) FUV/optical rest-frame spectrum of Ark 564 obtained by combining the FUSE spectrum (2001 June; 1000-1175 $\AA$ ), the HST G140L and G230L mean spectra (2000 May-July; 1175-3143 $\AA$ ), the Lick spectrum (1980; 3170-4160 $\AA$ ), and the Wise mean spectrum (1998 November-2001 January; 4160-7790 $\AA$ ). The FUSE spectrum has been scaled by a factor of 0.75 to match the flux level of the HST spectrum; the Wise spectrum has been corrected for the galaxy contribution $\left(F_{\text {gal }}=2.4 \times 10^{-15} \operatorname{ergs~s}^{-1} \mathrm{~cm}^{-2} \AA^{-1}\right.$; Shemmer et al. 2001). (b) Full spectrum after correction for reddening by using a standard Galactic extinction curve with $E(B-V)=0.03$ mag plus the intrinsic extinction curve that Crenshaw et al. (2002) derive for Ark 564 and $E(B-V)=0.14 \mathrm{mag}$. The data have been corrected for redshift $(z=0.02467$; de Vaucouleurs et al. 1991). The extinction-corrected spectrum $(b)$ has been offset by $1 \times 10^{-14} \mathrm{ergs} \mathrm{s}^{-1} \mathrm{~cm}^{-2} \AA^{-1}$ for clarity.

observed below $2 \mathrm{keV}$ was parameterized as a Gaussian of peak energy $E=0.57 \pm 0.02 \mathrm{keV}$ and mean equivalent width $(\mathrm{EW})=110_{-15}^{+11} \mathrm{eV}$. The soft hump component is also found to be variable in flux on timescales as short as 1 day and in shape on timescales as short as a few days (Paper I). Parameterization of the soft excess as a blackbody yields a temperature $T=1.8 \times 10^{6} \mathrm{~K}$ and luminosity $L_{\mathrm{bb}}=2.48 \times 10^{38} \mathrm{ergs} \mathrm{s}^{-1}$ (Paper I). A strong, ionized $(E \approx 7 \mathrm{keV}) \mathrm{Fe} \mathrm{K} \alpha$ line is detected, which shows variations in flux and $\mathrm{EW}$ on timescales as short as a week (Paper I).

The FUSE spectrum is dominated by the strong emission in the $\mathrm{O}$ VI $\lambda \lambda 1032,1038$ resonance doublet; heavily saturated absorption due to hydrogen Lyman-Werner bands, O vI, and $\mathrm{C}$ III are observed at velocities near the systemic redshift of Ark 564 (Paper V). The available data suggest that the UV and $\mathrm{X}$-ray absorbers are physically related, possibly identical, and spatially extended along the line of sight and characterized by total column density and ionization parameter $\log N_{\mathrm{H}}\left(\mathrm{cm}^{-2}\right) \approx$ 21 and $\log U \approx-1.5$. The absorbing gas is in a state of outflow with respect to the nucleus and carries out a kinetic luminosity about 1 order of magnitude smaller than the observed radiative luminosity of the source (Paper V).

Variations in the UV continuum flux (1365 A; Paper II) are well correlated with variations in both the hard $(2-10 \mathrm{keV})$ and soft $(0.75-2 \mathrm{keV}) \mathrm{X}$-ray fluxes and with the soft hump flux. No significant lags are detected between the variations in the X-ray and UV bands (Paper I). The variations of the continuum at $3000 \AA$ (Paper II) and at $4900 \AA$ (Paper III) lag behind those at
$1365 \AA$ by $\sim 1$ day and 1.8 days, respectively. These UV/optical delays were interpreted as evidence for a stratified continuum reprocessing region, possibly an accretion disk.

The variations of the Ly $\alpha$ emission line, which lag the variations of the continuum at $1365 \AA$ by 3 days, were used, in conjunction with the line width, to determine the virial mass of the central black hole, $M \lesssim 8 \times 10^{6} M_{\odot}$ (Paper II). This estimate is uncertain because of the low amplitude of the Ly $\alpha$ emission-line variations (1\%). However, the estimate in Paper II agrees with the one obtained by Pounds et al. (2001) based on a power spectrum analysis of X-ray variability. The black hole mass and $5100 \AA$ luminosity of Ark 564 are consistent with the hypothesis that NLS1s have lower black hole masses and higher accretion rates than BLS1s of comparable luminosity. The low-level variability observed in the emission lines is also different from most Seyfert 1 galaxies, which characteristically display variations of $10 \%$ on similar timescales.

\subsection{Reddening Correction in the Optical/FUV}

Given the indications (Paper IV) that strong intrinsic neutral absorption is present in Ark 564 in excess of the Galactic absorption, special care has been taken in correcting the data for reddening. We used a standard Galactic extinction curve with $E(B-V)=0.03$ mag plus the intrinsic extinction curve that Crenshaw et al. (2002 [Paper IV]) derive for Ark 564 and $E(B-V)=0.14 \mathrm{mag}$. The $H S T$ extinction correction was extrapolated linearly into the FUSE band, as suggested by Hutchings \& Giasson (2001) and Sasseen et al. (2002). The effect of reddening correction in the optical/UV bands is presented in Figure 2, where the observed spectrum in the $1000-7790 \AA$ wavelength region (labeled $a$ ) is compared to the absorption-corrected one (labeled $b$ ).

\section{THE INTRINSIC SED OF ARK 564}

Figure 3 shows the Ark 564 data in the IR/X-ray range. A power-law fit of the continuum in the optical/FUV region ${ }^{16}$ yields $F_{\lambda} \propto \lambda^{-1.58 \pm 0.01}$, hence spectral index $\alpha=0.42 \pm 0.01$ (specific flux $f_{\nu} \propto \nu^{-\alpha}$ ). Extrapolation of this power law in the $\mathrm{X}$-ray regime greatly overpredicts the X-ray flux (Fig. 3, dashed line). Analogously, the hard X-ray continuum slope $\left(\alpha_{A S C A}=\Gamma-1=1.538 \pm 0.005 ; \S 2.2\right.$; Fig. 3, long-dashdotted line) extrapolated to the lower energies overpredicts the optical/FUV flux, as previously noted by Walter \& Fink (1993). Clearly, both the optical/FUV and the X-ray power laws must break at some energy between the FUV and soft $\mathrm{X}$-ray. With the adopted reddening correction ( $\S 2.3$ ), the SED peaks at $\sim 50 \mathrm{eV}$.

Table 2 summarizes some relevant data from the SED derived using the simple parameterization of the combination of an optical/FUV power law with $\alpha=0.42$ breaking at $\sim 50 \mathrm{eV}$ to $\alpha_{A S C A}=1.538$ (hereafter SEDA), as described above, as well as the IRAS and IRTF data points ( $(2.1)$. Column (1) is the rest wavelength/energy; columns (2) and (3) list the observed and reddening-corrected values of $\nu L_{\nu}$, respectively. We

\footnotetext{
16 The fit was made to nine bands: $\lambda=1005-1007,1029.5-1030.5,1101-$ $1107,1114-1118,1155-1180,1350-1380,1460-1500,1620-1660$, and $7040-7050 \AA$. The uncertainties are purely statistical. The continuum fit in Paper II was performed only on the $H S T$ data corrected for Galactic reddening $[E(B-V)=0.06 \mathrm{mag}]$ and produced $F_{\lambda} \propto \lambda^{-0.88 \pm 0.01}$; this is comparable to our value for this reddening, $F_{\lambda} \propto \lambda^{-0.73 \pm 0.01}$, since the uncertainties are underestimated in both cases.
} 


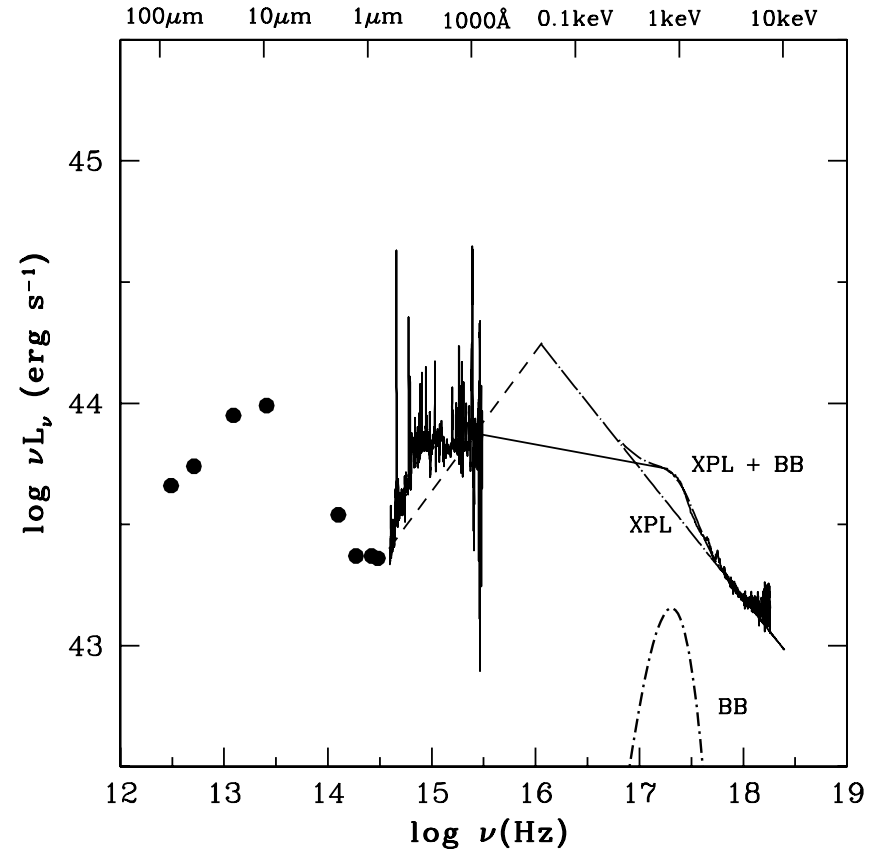

FIG. 3.-Reddening-corrected, rest-frame SEDs of Ark 564 (see $\S 3$ ). The optical/FUV data are the spectrum labeled $b$ in Fig. 2. The X-ray data are drawn from Paper I, while the IR data are from IRAS and IRTF ( $\S 2.1$ ). The shortdashed line shows the power-law fit of the optical/FUV data (spectral index $\alpha=0.42 \pm 0.01$ ) extrapolated to higher energies. The long-dash-dotted line is the power-law fit to hard X-ray data (XPL; $\alpha_{A S C A}=1.538 \pm 0.005 ; \S 2.2$ ) extrapolated to lower energies. The short-dash-dotted line is the blackbody model for the soft X-ray excess $(\mathrm{BB} ; \S 2.2)$. The solid line is a more conservative SED (SEDB) that connects the FUV and the soft X-ray data with a simple power law $(\alpha=1.08 ; \S 3)$.

estimate that the number of ionizing photons is $Q($ SEDA $) \approx$ $10^{55}$ photons s $^{-1}$.

We also considered a more conservative SED (Fig. 3, solid line, hereafter SEDB) in which the FUV and the soft X-ray data are connected with a simple power law $(\alpha=1.08)$, i.e, the combination of an optical/FUV power law with $\alpha=0.42$ breaking at $1000 \AA$ to $\alpha \approx 1$, then again breaking at $\approx 0.8 \mathrm{keV}$ to $\alpha_{A S C A}=1.538$. The number of ionizing photons for SEDB is $Q(\mathrm{SEDB}) \approx 5 \times 10^{54}$ photons $\mathrm{s}^{-1}$, which is consistent with what Crenshaw et al. (2002) found. ${ }^{17}$

\section{PHOTOIONIZATION MODELING}

In this section, we address the issue of the energy budget of Ark 564 by examining the emission lines observed in its spectrum, and we deduce the mean physical properties of the line-emitting gas, hydrogen density $n$ and ionization parameter $U\left(U=Q / 4 \pi r^{2} n c, r\right.$ being the distance to the ionizing source and $c$ the speed of light) through photoionization modeling given the assumed SEDs. We expect a range of ionization to exist throughout the broad-line region (BLR), to accurately describe which multizone modeling would be required; however, here we use a single density and ionization parameter modeling to derive the mean BLR properties. For each of the input continua $(\S 4.2)$, we considered a total hydrogen density of $n=10^{9}, 10^{11}, 10^{12}$, and $10^{13} \mathrm{~cm}^{-3}$ and calculated the predicted intensities of the major emission lines for a range of ionization parameters between $\log U=-4$ and 2 . In the case of "table AGN" ( $\S 4.2$ ), we specified a grid of $n$ and $U$ values.

\footnotetext{
17 Note that SEDB corresponds to SED2 in Paper V.
}

For the other input continua, we normalized the SEDs with respect to the measured X-ray luminosity in the absorptioncorrected rest-frame $2-10 \mathrm{keV}$ energy range $\left(L_{2}-10 \mathrm{keV}=\right.$ $2.4 \times 10^{43}$ ergs $\mathrm{s}^{-1}$; Paper I) and specified the radius of the cloud, thus obtaining $U$. In the case of SEDA and SEDB, using an observed SED assumes that the gas responsible for the emission lines sees the same ionizing continuum as the observer does; therefore, we expect SEDA and SEDB to yield more realistic predictions of the emitted line spectrum.

\subsection{Emission-Line Fluxes}

The fluxes (relative to $\mathrm{H} \beta$ ) of the most prominent emission lines in the 1150-6817 $\AA$ wavelength range have been published in Table 2 of Paper IV; here we report a selection of them in Table 3 (col. [2], relative to $\operatorname{Ly} \alpha$ ). Because of the NLS1 nature of Ark 564, the contributions from the broad emission line region (BELR) and narrow emission line region (NELR) are strongly blended together and those line ratios include both components. The measured fluxes were corrected for reddening using the continuum reddening curve (Crenshaw et al. 2002), given the similar extinctions for the continuum and emission lines; the errors are propagated in quadrature from the ones listed in Paper IV, and they include photon noise, continuum placement errors, and reddening errors.

Columns (2) and (3) of Table 3 also report the fluxes of C III $\lambda 977$ and the $\mathrm{O}$ vi $\lambda \lambda 1032,1038$ doublet, which can help better constrain the value of $n$ and $U$. These lines were modeled in Paper V; hence, both a broad and a narrow component are

TABLE 2

Data from the Spectral Energy Distribution

\begin{tabular}{|c|c|c|}
\hline $\begin{array}{c}\text { Rest Wavelength/Energy } \\
\text { (1) }\end{array}$ & $\begin{array}{c}\nu L_{\nu}(\text { Observed }) \\
\left(\times 10^{43} \text { ergs s }^{-1}\right) \\
(2)\end{array}$ & $\begin{array}{c}\nu L_{\nu}(\text { Intrinsic, SEDA }) \\
\left(\times 10^{43} \mathrm{ergs} \mathrm{s}^{-1}\right) \\
(3)\end{array}$ \\
\hline $97.59 \mu \mathrm{m}^{\mathrm{a}} \ldots$. & 3.98 & 4.57 \\
\hline $58.56 \mu \mathrm{m}^{\mathrm{a}} \ldots$. & 4.82 & 5.50 \\
\hline 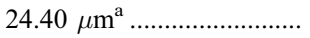 & 7.91 & 8.91 \\
\hline 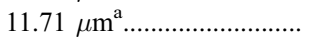 & 8.73 & 9.77 \\
\hline $2.4 \mu \mathrm{m}^{\mathrm{b}} \ldots \ldots$ & 3.3 & 3.5 \\
\hline $1.6 \mu \mathrm{m}^{\mathrm{b}} \ldots \ldots$ & 2.2 & 2.4 \\
\hline $1.14 \mu \mathrm{m}^{\mathrm{b}} \ldots$ & 2.1 & 2.4 \\
\hline $1 \mu \mathrm{m}^{\mathrm{c}}$ & 2.045 & 2.165 \\
\hline $9850 \AA^{\mathrm{b}} \ldots \ldots$ & 2.0 & 2.3 \\
\hline $7000 \AA$ & 1.851 & 2.660 \\
\hline 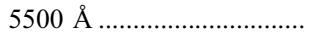 & 1.73 & 3.057 \\
\hline 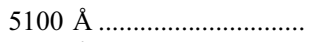 & 1.694 & 3.193 \\
\hline $3000 \AA \AA$ & 1.461 & 4.338 \\
\hline 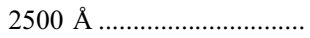 & 1.388 & 4.819 \\
\hline 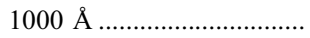 & 1.075 & 8.178 \\
\hline $0.046 \mathrm{keV}^{\mathrm{d}} \ldots \ldots \ldots \ldots \ldots \ldots \ldots \ldots \ldots \ldots \ldots \ldots \ldots$ & $\ldots$ & 17.5 \\
\hline 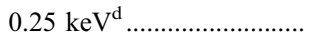 & $\ldots$ & 7.195 \\
\hline $0.78 \mathrm{keV} \ldots \ldots \ldots \ldots \ldots \ldots \ldots \ldots$ & 2.895 & 5.240 \\
\hline $1 \mathrm{keV}$ & 3.046 & 4.668 \\
\hline $2 \mathrm{keV}$ & 2.060 & 2.419 \\
\hline $10 \mathrm{keV}$ & 0.942 & 0.970 \\
\hline
\end{tabular}

Notes.-The intrinsic optical/UV/X-ray data are from the reddeningcorrected, rest-frame SEDA $(\S 3)$. SEDB is SEDA with the exclusion of the point at $\sim 50 \mathrm{eV}$. We adopt $H_{0}=75 \mathrm{~km} \mathrm{~s}^{-1} \mathrm{Mpc}^{-1}, q_{0}=0.5$.

a IRAS data points $(\S 2.1)$.

b IRTF data points $(\S 2.1)$

c Extrapolated value from the optical/FUV power law (spectral index $\alpha=0.42 \pm 0.01 ; \S 3)$.

d Extrapolated value from the $A S C A$ power law $\left(\alpha_{A S C A}=1.538 \pm 0.005\right.$; $\S 2.2)$, peak of SEDA ( $§ 3)$. 
TABLE 3

Arakelian 564 Emission-Line Characteristics

\begin{tabular}{|c|c|c|c|c|c|c|}
\hline $\begin{array}{l}\text { Line } \\
(1)\end{array}$ & $\begin{array}{c}F / F(\mathrm{Ly} \alpha) \mathrm{BEL}+\mathrm{NEL}^{\mathrm{a}} \\
(2)\end{array}$ & $\begin{array}{c}F / F(\operatorname{Ly} \alpha) \mathrm{BEL}^{\mathrm{a}} \\
\text { (3) }\end{array}$ & $\begin{array}{c}F / F(\mathrm{Ly} \alpha) \mathrm{QSO}^{\mathrm{b}} \\
\text { (4) }\end{array}$ & $F / F(\operatorname{Ly} \alpha) \underset{(5)}{\mathrm{NGC}} 5548^{\mathrm{c}}$ & $\begin{array}{c}\text { FWHM } \\
\left(\mathrm{km} \mathrm{s}^{-1}\right)^{\mathrm{d}} \\
(6)\end{array}$ & $\begin{array}{c}\text { References } \\
\text { (7) }\end{array}$ \\
\hline C III $\lambda 977$...................... & $0.053 \pm 0.017$ & $0.031 \pm 0.015$ & $\ldots$ & $\ldots$ & 4000,1100 & 1,2 \\
\hline 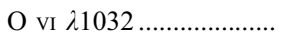 & $0.284 \pm 0.032$ & $0.200 \pm 0.052$ & $\ldots$ & 0.036 & 4000,1100 & 1,2 \\
\hline O vi $\lambda 1038 \ldots \ldots \ldots \ldots \ldots \ldots$ & $0.141 \pm 0.048$ & $0.100 \pm 0.026$ & $\ldots$ & 0.018 & 4000,1100 & 1,2 \\
\hline O vi $\lambda 1032,1038 \ldots \ldots \ldots . .$. & $0.425 \pm 0.141$ & $0.300 \pm 0.076$ & $0.1-0.3^{\mathrm{e}}$ & 0.054 & 4000,1100 & 1,2 \\
\hline $\operatorname{Ly} \alpha \lambda 1216 \ldots \ldots \ldots \ldots \ldots \ldots$ & 1.00 & 1.00 & 1.00 & 1.00 & 2114 & 3,4 \\
\hline $\mathrm{N}$ v $\lambda 1240 \ldots \ldots \ldots \ldots \ldots \ldots . . . . . . .$. & $0.275 \pm 0.118$ & $\ldots$ & $0.09-0.26$ & 0.119 & 2809 & 3,4 \\
\hline 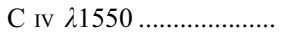 & $0.215 \pm 0.087$ & $\ldots$ & $0.28-0.42$ & 0.937 & 1934 & 3,4 \\
\hline 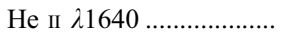 & $0.101 \pm 0.040$ & $\ldots$ & $0.06-0.13^{\mathrm{f}}$ & $0.143^{\mathrm{f}}$ & 1195,1831 & 3,4 \\
\hline O III] $\lambda 1663 \ldots \ldots \ldots \ldots \ldots \ldots$ & $0.038 \pm 0.015$ & $\ldots$ & $\ldots$ & $\ldots$ & $\ldots$ & 3,4 \\
\hline 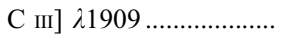 & $0.068 \pm 0.026^{\mathrm{g}}$ & $\ldots$ & $0.09-0.19^{\mathrm{h}}$ & $0.171^{\mathrm{g}}$ & 1920 & 3,4 \\
\hline Mg II $\lambda 2800 \ldots \ldots \ldots \ldots \ldots \ldots$ & $0.066 \pm 0.008$ & $\ldots$ & $0.06-0.13$ & 0.188 & 1659 & 3,5 \\
\hline 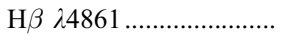 & $0.063 \pm 0.019$ & $\ldots$ & $0.02-0.05$ & $\ldots$ & 700 & 3 \\
\hline 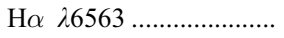 & $0.240 \pm 0.080$ & $\ldots$ & $0.05-0.09^{\mathrm{i}}$ & $\ldots$ & $\ldots$ & 3 \\
\hline
\end{tabular}

a Reddening-corrected flux relative to Ly $\alpha$ derived from Crenshaw et al. 2002 . The lines are corrected by using $E(B-V)=0.14 \pm 0.04$ mag and the Ark 564 reddening curve from Crenshaw et al. 2002 plus $E(B-V)=0.03$ mag and the Galactic curve $(\S 2.3)$.

$\mathrm{b}$ Data from the mean observed QSO spectrum in Baldwin et al. 1995 and references therein, corrected for the reddening appropriate for Ark 564.

c BEL fluxes, corrected for NEL contribution and Galactic reddening of the Seyfert 1.5 NGC 5548; derived from Korista \& Goad 2000 and references therein. The $\mathrm{O}$ vi line ratios are derived from the BEL values in Brotherton et al. 2002, then corrected for Galactic reddening $[E(B-V)=0.03$ mag and extinction law of Cardelli, Clayton, \& Mathis 1989].

${ }^{\mathrm{d}}$ Model FWHM of C III $\lambda 977$ and O vi relative to BEL and NEL components (Paper V), separately; the others are measured on the whole line profile (Paper II). The He II values are relative to the G140L and G230L mean spectrum, respectively (Paper II).

e Total O vi + Ly $\beta$ flux. This compares with $0.434 \pm 0.141$ for Ark 564 .

${ }^{\mathrm{f}}$ Total He II $+\mathrm{O}_{\text {III] }} \lambda 1666$ flux. This compares with $0.139 \pm 0.043$ for Ark 564 .

g Total C III] $\lambda 1909+$ Si III] $\lambda 1892$ flux.

$\mathrm{h}$ Total C III] $\lambda 1909+\mathrm{Si}$ III] $\lambda 1892+\mathrm{Al}$ III $\lambda 1990$ flux.

${ }^{\mathrm{i}}$ Based on the range of values of $\mathrm{H} \alpha / \mathrm{H} \beta$ (3.97-6.64) from the NLS1 sample of Osterbrock \& Pogge 1985.

References.-(1) This work; (2) Paper V; (3) Paper IV; (4) Paper II; (5) Paper III.

available (denoted with BEL and NEL, respectively; cols. [2] and [3]). In order to compare with the other line ratios, we needed to account for the different contribution of BELR and NELR gas to $H \beta$, which we estimated as follows. We assumed that the ratio of the narrow component of $\mathrm{H} \beta$ and [O III] $\lambda 5007$ in NGC 5548, i.e., $0.12 \pm 0.01$ (Kraemer et al. 1998), can be used for Ark 564; we scaled it to the observed [O III] $\lambda 5007$ flux for Ark 564, $F\left([\mathrm{O}\right.$ III] 25007$)=(2.4 \pm 0.1) \times 10^{13} \mathrm{ergs}$ $\mathrm{s}^{-1} \mathrm{~cm}^{-2}$ (Paper III), obtaining the NEL ratio $F(\mathrm{H} \beta) / F([\mathrm{O}$ III $]$ 25007) in Ark 564. Thus, we estimated that roughly $75 \%$ of the total $\mathrm{H} \beta$ flux is from the BEL and bracketed this value between $50 \%$ and $100 \%$, given that the BEL and NEL contributions to $\mathrm{C}_{\text {III }} \lambda 977$ and $\mathrm{O}$ VI $\lambda \lambda 1032,1038$ are the same and that the total flux is the absolute upper limit to the BEL flux. We note that Rodríguez-Ardila et al. (2000) found that on average, $50 \%$ of the flux of the total $\mathrm{H} \beta$ is due to emission from the NELR and that the $F\left(\left[\mathrm{O}_{\text {III }}\right] \lambda 5007\right) / F(\mathrm{H} \beta)$ emitted in the NELR varies from 1 to 5 , which is much lower than our adopted value $(\sim 8.3)$. This result is sustained by the analysis of Contini, Rodríguez-Ardila, \& Viegas (2003). However, Véron-Cetty, Véron, \& Gonçalves (2001) point out that the low $F([\mathrm{O}$ III $] 25007) / F(\mathrm{H} \beta)$ values found by Rodríguez-Ardila et al. (2000) for NLS1s are due to the fact that they modeled the broad Balmer component with a Gaussian rather than a Lorentzian. In the analysis of Veron-Cetty et al. (2001) the $F([\mathrm{O}$ III] 25007$) / F(\mathrm{H} \beta)$ ratios span the range measured in broad-line Seyfert galaxies, which makes our use of a value derived from a well-studied BLS1 reasonable. Furthermore, Nagao, Murayama, \& Taniguchi (2001) also show that $F([\mathrm{O}$ I $]$ $\lambda 6300) / F([\mathrm{O}$ III $] \lambda 5007)$ and $F([\mathrm{O}$ III $] \lambda 4363) / F([\mathrm{O}$ III $] \lambda 5007)$ are indistinguishable in NLS1s and BLS1s. Given this ambiguity, in the following analysis we will note where our assumptions for the deconvolution of $\mathrm{O}$ VI $\lambda \lambda 1032,1038$ and C III $\lambda 977$ affect the results.

As a comparison, column (4) of Table 3 reports the corresponding values for a mean QSO spectrum, which we derived from Baldwin et al. (1995) by applying the reddening correction appropriate for Ark 564; the $\mathrm{H} \alpha / \mathrm{Ly} \alpha$ ratio is derived from Osterbrock \& Pogge (1985). Column (5) lists the BEL fluxes of the Seyfert 1.5 NGC 5548, corrected for NEL contribution and Galactic reddening (Korista \& Goad 2000 and references therein). Column (6) lists the FWHM of the lines, as drawn from Papers V, II, and III (C III $\lambda 977$ and O VI are the model BEL and NEL components, while the others are measured on the whole line profile). All errors are propagated in quadrature. Finally, column (7) reports the references for columns (2), (3), and (6). Table 3 shows that carbon in Ark 564 is at the lower end and nitrogen at the upper end of the mean QSO distribution, and some interesting differences can be found with respect to the Seyfert 1.5 NGC 5548. Indeed, N v $\lambda 1240$ is stronger in Ark 564 by a factor of $\sim 2.3$, while C IV $\left.\lambda 1550, \mathrm{C}_{\text {III }}\right] \lambda 1909$, and $\mathrm{Mg}$ II $\lambda 2800$ are weaker in Ark 564 by factors of $\sim 4.4,2.5$, and 2.8 , respectively.

\subsection{Input Continua}

We used the code CLOUDY ${ }^{18}$ (ver. 94.00; Ferland 1996) to predict the intensities of the lines produced by the BEL gas through photoionization modeling. Our choices of input continua for CLOUDY are shown in Figure 4. In brief, these are

1. The CLOUDY "table AGN" continuum, which is the Mathews \& Ferland (1987) continuum modified with a

\footnotetext{
18 http://www.nublado.org.
} 


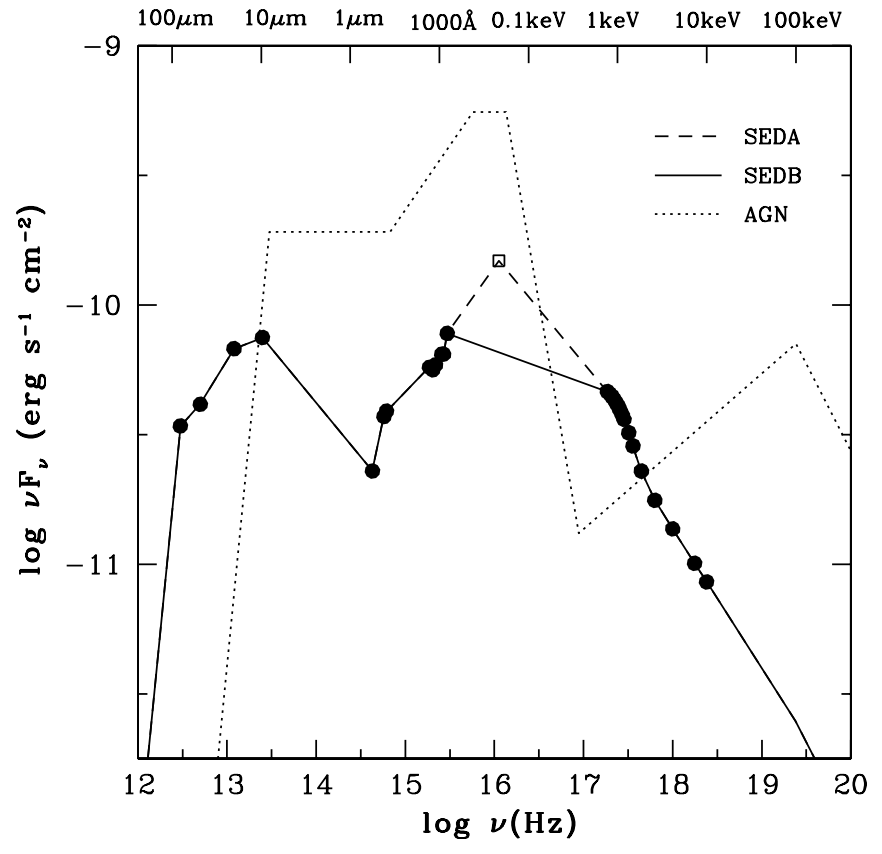

FIG. 4.- Input SEDs for CLOUDY, normalized to the absorption-corrected rest-frame flux at $2 \mathrm{keV}$. The dotted line is the "table AGN" model in CLOUDY; the dashed line is the SEDA described in $\S 3$ (the circles are the observed points, the square the extrapolated point); the solid line is the conservative SEDB described in $\S 3$.

submillimeter break at $10 \mu \mathrm{m}$, so that the spectral index is changed from -1 to $-5 / 2$ for frequencies below the millimeter break.

2. The SEDA input continuum, which was created from points chosen from the SED presented in $\S 3$ (Fig. 4, circles), as well as one extrapolated point (Fig. 4, square), i.e., where the optical/FUV and X-ray power-law extrapolations meet. In particular, in the X-ray, we used continuum points from the power-law fit and added a blackbody component (Fig. 3, dotdashed line) of temperature $T=1.8 \times 10^{6} \mathrm{~K}$ and luminosity $L_{\mathrm{bb}}=2.48 \times 10^{38} \mathrm{ergs} \mathrm{s}^{-1}(\S 2.2)$.

3. The more conservative SEDB input continuum, which uses only points from the observed SED (Fig. 4, circles only).

\subsection{Physical Conditions of the Emission-Line Gas}

Figure 5 shows the line intensity of $\mathrm{C}$ III] $\lambda 1909$ relative to Ly $\alpha$ as a function of hydrogen density $\left[\log n\left(\mathrm{~cm}^{-3}\right)=9,11\right.$, $12,13]$ and input continuum ("table AGN", SEDA, and SEDB discussed in $\S 4.2)$. The horizontal lines correspond to the observed value of C III] $\lambda 1909$ and its errors listed in Table 3. Solutions to $U$ (as also shown in Figs. 6 and 7) are double valued, but we prefer higher values based on line widths, as discussed below. As expected for a semiforbidden transition, the $\left.\mathrm{C}_{\mathrm{III}}\right] \lambda 1909$ line becomes collisionally suppressed as the density increases, arguing for an upper limit for the density of $\log n \lesssim 12$. Analogously, Figure 6 shows the intensity of C III $\lambda 977$ and total $\mathrm{N} \vee \lambda 1240$, which indicate $\log n>9$. Furthermore, the observed $\mathrm{C}$ III] $\lambda 1909$ implies $\log U \approx-3.3$ or $\log$ $U \approx-0.7$ for $\log n=9$, both of which are much lower than the values required for $\mathrm{O}$ vI, for which we derive $\log U \approx-1.1$ or $0.8^{19}$ (from a plot analogous to Fig. $7 b$ relative to $\log n=9$ ); i.e., no consistent result can be found for low densities.

\footnotetext{
${ }^{19}$ We find $\log U \approx-0.9$ or 0.8 if we do not deconvolve BEL and NEL.
}

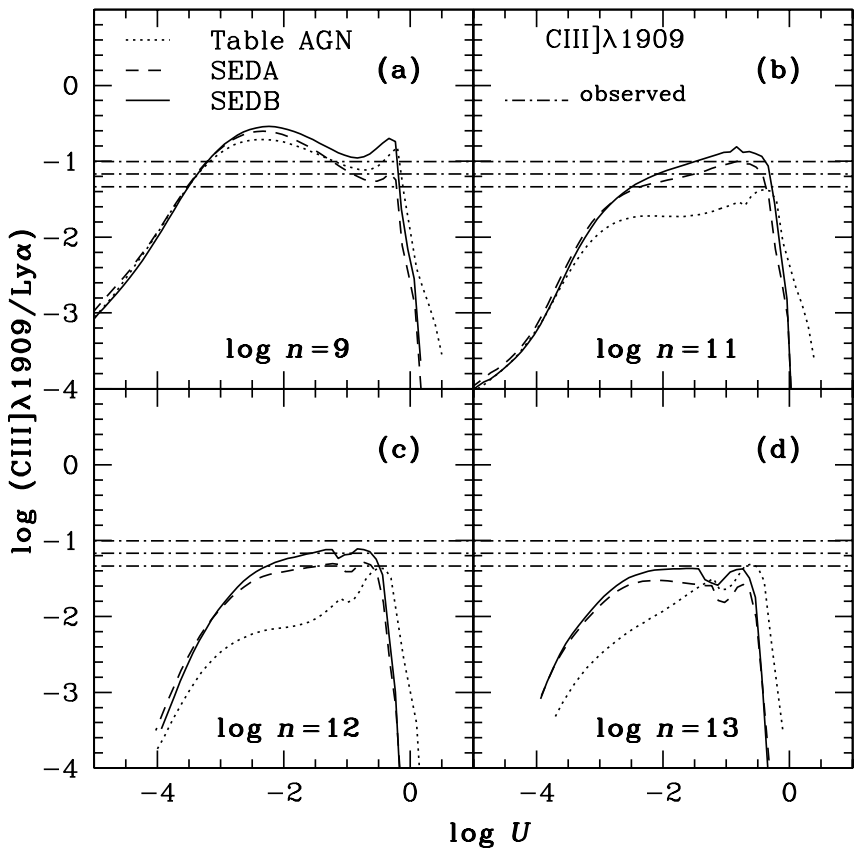

FIG. 5.- Line intensity relative to Ly $\alpha$ of $\mathrm{C}$ III] $\lambda 1909$ as a function of hydrogen density $(\log n=9,11,12,13)$ and input continuum (table AGN SEDA, and SEDB discussed in $\S 4.2$ ). The horizontal dash-dotted lines correspond to the observed values and their errors.

Considering $\log n \approx 11$ as a plausible estimate of the density, we can investigate the value of the ionization parameter. This has always been a difficult problem because with only optical/UV observations, multiple ionization states of a single element are not observed, so the values of $U$ are highly model dependent. With the multiwavelength, multimission observations of Ark 564, we now have observations of both C III (with FUSE) and C Iv (with HST), so we can actually measure the ionization parameter. As shown in Figure $7 a$, the $\mathrm{C}$ III $\lambda 977 / \mathrm{C}$ IV $\lambda 1550$ ratio constrains the ionization parameter to

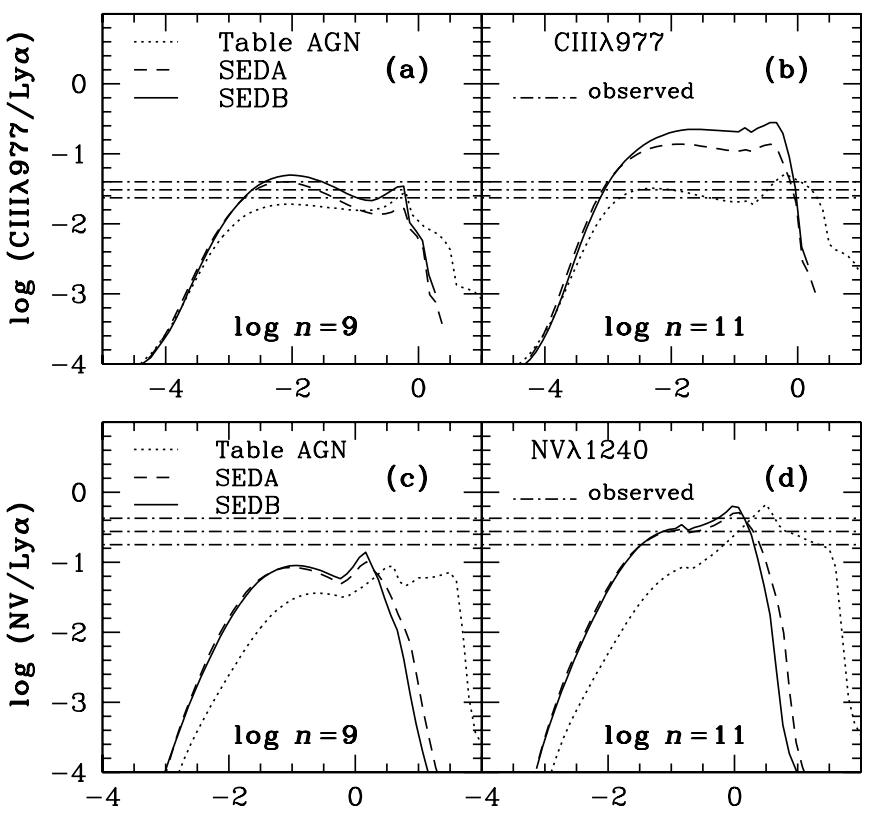

FIG. 6.-Same as Fig. 5 for $\mathrm{C}$ III $\lambda 977$ (top) and N v $\lambda 1240$ (bottom) for $\log n=9$ and 11 . 


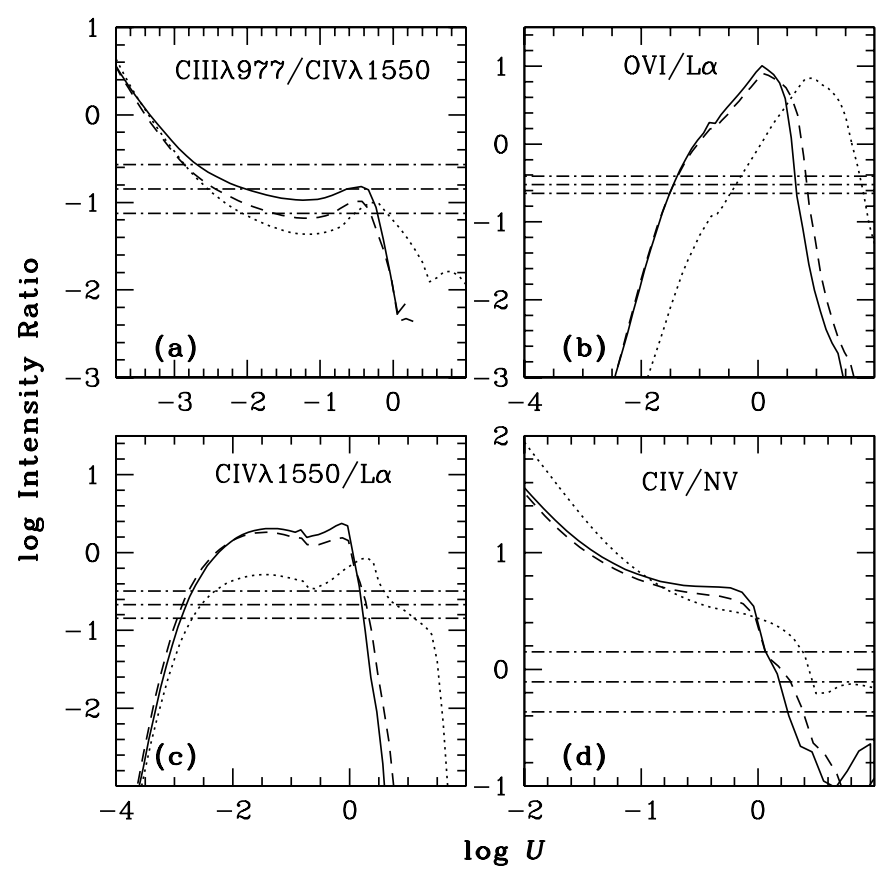

FIG. 7.- Line intensity ratios of (a) $\mathrm{C}$ III $\lambda 977 / \mathrm{C}$ IV $\lambda 1550,(b) \mathrm{O}$ vI/Ly $\alpha$, (c) $\mathrm{C}$ IV $\lambda 1550 / \mathrm{Ly} \alpha$, and $(d) \mathrm{C}$ IV $\lambda 1550 / \mathrm{N} \vee \lambda 1240$ as a function of input continuum (same line notations as in Fig. 5). For all cases, $\log n=11$.

$\log U=[-2.88,-0.22] .{ }^{20}$ Studies of reverberation mapping have shown that the BLR is stratified (Peterson \& Wandel 1999), so a single value of $U$ cannot possibly correspond to the entire BLR, but the range of $U$ determined above must be the dominant range of $U$ where $\mathrm{C}$ IV emission is produced. The higher ionization lines, e.g., $\mathrm{O}$ VI $\lambda \lambda 1032,1038$ and $\mathrm{He}$ II $\lambda 1640$, are likely to be produced closer in with higher ionization parameter. Indeed, as shown in Figures $7 b$ and $6 d$, somewhat higher values of $U$ are preferred for $\mathrm{O}$ VI $\left(\log U=[-1.52,-1.36]\right.$ or $\left.\log U=[0.61,0.87]^{21}\right)$ and $\mathrm{N} \mathrm{v}$ $(\log U=[-1.43,0.4])$.

Figure $7 c$ shows the $C$ IV $\lambda 1550$ intensity ratio and implies that either $\log U=[-2.94,-2.66]$ or $\log U=[0.18,0.34]$. Figure $7 d$ shows the intensity ratio $\mathrm{C}$ IV $\lambda 1240 / \mathrm{N} v \lambda 1550$, for which we derive $\log U=[0.07,0.39]$. The comparison with the BLS1 NGC 5548 shows that N v is stronger in Ark 564 by a factor of $\sim 2.3$, while $\mathrm{C}$ IV is weaker in Ark 564 by a factor of $\sim 4.4$ ( $\{4.1$ and Table 3 ). Hence, the observed $\mathrm{C}$ IV/ $\mathrm{N} \mathrm{v}$ ratio may be roughly 1 order of magnitude smaller in Ark 564, and the observed limits in Figure $7 d$ probably reflect an overabundance of $\mathrm{N}$ (or $\mathrm{C}$ depletion).

To discriminate between the $\operatorname{low}-U(\log U \approx-1.5)$ and high- $U(\log U \approx 0)$ solutions, we derive the distance of the BELR gas from $R_{\mathrm{BELR}}=(Q / 4 \pi c n U)^{1 / 2} \approx 1.63 \times$ $10^{16}\left(Q_{55} / U\right)^{1 / 2} n_{11}^{-1 / 2} \mathrm{~cm}$, where $Q_{55}=Q / 10^{55}$, assuming the above values of $U$ and $n$, and the photon luminosity $Q$ from $\S 3$. For $\log n=11$ and $\log U \approx-1.5$, the inferred distance of the BELR is $R_{\mathrm{BELR}}^{\mathrm{SEDA}}=9.2 \times 10^{16} \mathrm{~cm}$ for $Q(\mathrm{SEDA})$ and $R_{\mathrm{BELR}}^{\mathrm{SEDB}}=6.5 \times 10^{16} \mathrm{~cm}$ for $Q(\mathrm{SEDB})$. For $\log n=11$ and $\log U \approx 0, \quad R_{\mathrm{BELR}}^{\mathrm{SEDA}}=1.63 \times 10^{16} \mathrm{~cm}$ and $R_{\mathrm{BELR}}^{\mathrm{SEDB}}=1.15 \times$ $10^{16} \mathrm{~cm}$. For a central mass of $M=8 \times 10^{6} M_{\odot}$, the expected velocity dispersion (Peterson et al. 2000) is $V=$ $\left(G M / R_{\mathrm{BLR}} f\right)^{0.5}$, with $f=3 / \sqrt{2}$. This corresponds to $740-$

\footnotetext{
${ }^{20} \log U=[-3.07,-0.36]$ if we do not deconvolve BEL and NEL.

${ }^{21} \log U=[-1.44,-1.23]$ or $\log U=[0.64,0.79]$ if we do not deconvolve BEL and NEL.
}

$880 \mathrm{~km} \mathrm{~s}^{-1}$ for the low- $U$ solutions and $1750-2090 \mathrm{~km} \mathrm{~s}^{-1}$ for the high- $U$ solutions. Column (6) of Table 3 shows that FWHM $($ C IV $)=1934 \mathrm{~km} \mathrm{~s}^{-1}$, FWHM $(\mathrm{C} \mathrm{III})=1920 \mathrm{~km} \mathrm{~s}^{-1}$, and FWHM $(\mathrm{N} \mathrm{V})=2809 \mathrm{~km} \mathrm{~s}^{-1}$. Therefore, the comparison with the observed FWHMs favors the high- $U$ solutions. Finally, Table 3 shows that the FWHM of O vI is larger than that of $\mathrm{N} \mathrm{v}$, which is larger than that of $\mathrm{C}$ IV, again consistent with the stratified BLR model.

\section{DISCUSSION}

A nonsimultaneous optical, UV, and X-ray SED of Ark 564 was presented by Comastri et al. (2001), who found that it peaks in the soft X-ray band. Here we present a spectral energy distribution that is obtained from contemporaneous data covering almost 5 decades in energy. ${ }^{22}$ Simultaneity is particularly important for NLS1s, since, as a class, they are extremely variable in time, although Ark 564 has shown only weak variability in the optical/UV bands (Papers II and III).

We report some relevant data from the SED in Table 2. These were derived using the simple parameterization of the combination of an optical/FUV power law with spectral index $\alpha=0.42$ breaking at $\sim 50 \mathrm{eV}$ to $\alpha_{A S C A}=1.538$ (SEDA in Fig. 3), as well as the IRAS and IRTF data points (§ 2.1). A more conservative SED, instead, connects the FUV and the soft X-ray data with a simple power law $(\alpha=1.08$; Fig. 3, solid line) and is a combination of an optical/FUV power law with $\alpha=0.42$ breaking at $1000 \AA$ to $\alpha=1.081$, then again breaking at $\approx 0.8 \mathrm{keV}$ to $\alpha_{A S C A}=1.538$ (SEDB in Fig. 3 ). The ambiguity in the shape of the SED in the $900 \AA$ to $0.8 \mathrm{keV}$ region is rather unfortunate, since a considerable portion of the energy of Ark 564 might be output in this range. Previous ROSAT (Brandt et al. 1994) and BeppoSAX (Comastri et al. 2001) data showed a flattening of the soft excess toward the lowest X-ray energies available, and the $X M M$ spectrum obtained during the monitoring campaign of 2000 shows a definite curvature in the soft excess (Vignali et al. 2004).

An interesting issue is how Ark 564 compares with other NLS1 galaxies and with BLS1s in terms of its broadband properties, as they can be quantified by spectral indices. Table 4 reports the intrinsic spectral indices calculated between different wavelength bands for Ark 564 and, as a comparison, the corresponding values for the NLS1 Ton S180 and BLS1s. These show that while the interband properties of Ton S180 are not significantly different from the ones observed in BLS1s (Turner et al. 2002), this may not be the case for Ark 564. Table 4 indicates that the two NLS1s have steeper X-ray slopes than BLS1s, Ark 564 more so than Ton $\mathrm{S} 180\left(\alpha_{\mathrm{x}}=1.57\right.$ and 1.44, respectively, compared with 0.91 for BLS1s), which is consistent with the general characteristics of NLS1s. The optical/ $\mathrm{X}$-ray spectral index $\left(\alpha_{\text {ox }}\right)$, on the other hand, is lower in Ark 564 than in BLS1s and Ton S180, with $\alpha_{\text {ox }}=1.11$ for Ark $564^{23}$ and 1.52 for Ton S180. This reflects the fact that Ark 564 is relatively more X-ray-bright, or that the optical continuum is suppressed, compared to other AGNs. The other indices also reflect the X-ray brightness of Ark 564 when compared with BLS1s. We cannot exclude the possibility that intrinsic reddening in excess of the one considered here may be

\footnotetext{
22 The Lick spectrum, which was obtained in 1980, is merely used to fill in a small gap in the optical data, and the 2001 FUSE spectrum shows a continuum slope consistent with the one obtained from the $H S T$ spectrum. This latter fact suggests that, although the flux level changed between 2000 and 2001 , the overall shape of the optical/FUV SED did not change.

${ }^{23}$ The observed $\alpha_{\text {ox }}$ is 0.94 in Ark 564.
} 
TABLE 4

Spectral Indices

\begin{tabular}{|c|c|c|c|c|c|}
\hline $\begin{array}{l}\text { Index } \\
\text { (1) }\end{array}$ & $\begin{array}{l}\text { Definition } \\
\text { (2) }\end{array}$ & $\begin{array}{c}\text { Ark } 564^{\mathrm{a}} \\
\text { (3) }\end{array}$ & $\begin{array}{c}\text { Ton } S 180^{\mathrm{a}} \\
\text { (4) }\end{array}$ & $\begin{array}{l}\text { BLS1 } \\
\text { (5) }\end{array}$ & $\begin{array}{l}\text { References } \\
\text { (6) }\end{array}$ \\
\hline 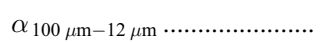 & $-1.086 \log \left(F_{12 \mu \mathrm{m}} / F_{100 \mu \mathrm{m}}\right)$ & 0.64 & $\ldots$ & $\ldots$ & \\
\hline 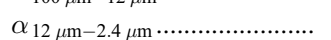 & $-1.431 \log \left(F_{2.4 \mu \mathrm{m}} / F_{12 \mu \mathrm{m}}\right)$ & 1.64 & $\ldots$ & $\ldots$ & \\
\hline 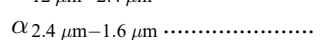 & $-5.679 \log \left(F_{1.6 \mu \mathrm{m}} / F_{2.4 \mu \mathrm{m}}\right)$ & 1.94 & $\ldots$ & $\ldots$ & \\
\hline 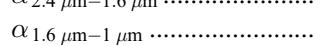 & $-4.900 \log \left(F_{1 \mu \mathrm{m}} / F_{1.6 \mu \mathrm{m}}\right)$ & $1.18^{\mathrm{b}}$ & $\cdots$ & $\cdots$ & \\
\hline 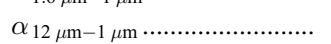 & $-0.927 \log \left(F_{1 \mu \mathrm{m}} / F_{12 \mu \mathrm{m}}\right)$ & $1.60^{\mathrm{b}}$ & & & \\
\hline$\alpha_{3000 \AA-1000 \AA}\left(\alpha_{\text {uV }}\right) \ldots \ldots \ldots \ldots$. & $-2.096 \log \left(F_{1000 \AA} / F_{3000 \AA}\right)$ & 0.42 & 0.66 & 1.25 & 1 \\
\hline 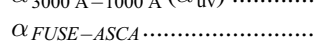 & c & 1.08 & $\ldots$ & $\ldots$ & 1 \\
\hline$\alpha_{5500 \AA-0.25 \mathrm{keV}} \ldots \ldots \ldots \ldots \ldots \ldots$ & $-0.489 \log \left(F_{0.25 \mathrm{keV}} / F_{5500 \AA}\right)$ & $0.82^{\mathrm{b}}$ & 1.12 & 0.73 & 2 \\
\hline$\alpha_{5500 \AA-1 \mathrm{keV}}\left(\alpha_{\text {ox-hard }}\right) \ldots \ldots$. & $-0.378 \log \left(F_{1 \mathrm{keV}} / F_{5500 \AA}\right)$ & 0.93 & 1.38 & 1.13 & 3 \\
\hline 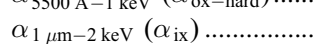 & $-0.312 \log \left(F_{2 \mathrm{keV}} / F_{1 \mu \mathrm{m}}\right)$ & $1.01^{\mathrm{b}}$ & 1.35 & $1.14-2.16$ & 4 \\
\hline$\alpha_{2500 \AA-2 \mathrm{keV}}\left(\alpha_{\text {ox }}\right) \ldots \ldots \ldots \ldots . . . . . .$. & $-0.384 \log \left(F_{2 \mathrm{keV}} / F_{2500 \AA}\right)$ & 1.11 & 1.52 & $1.46_{-0.07}^{+0.05}, 1.21 \pm 0.02$ & 5,6 \\
\hline$\alpha_{\mathrm{x}}$ & $-1.431 \log \left(F_{10 \mathrm{keV}} / F_{2 \mathrm{keV}}\right)$ & 1.57 & 1.44 & 0.91 & 7 \\
\hline
\end{tabular}

NoтE.-For spectral indices relative to the Ark 564 IRAS points we used the reddening-corrected, redshift-corrected fluxes and wavelengths

a Intrinsic, i.e., reddening-corrected, redshift-corrected (SEDA).

b Based on extrapolated value (§ 3, Fig. 3).

c Simple power law connecting the high-energy end of the FUSE spectrum and the low-energy end of the ASCA spectrum ( $\S 3$, Fig. 3).

REFERENCES.-(1) Cheng, Gaskell, \& Koratkar 1991; index in the 2200-1200 $\AA$ band, based on the BLS1 subsample; (2) Turner et al. 1999a; (3) Grupe et al. 1998; (4) Lawrence et al. 1997; (5) Zamorani et al. 1981; (6) Puchnarewicz et al. 1996; (7) Nandra et al. 1997b.

present that could be suppressing the optical, but we argue that it would require the continuum to be more reddened than the emission lines (e.g., He II $\lambda 1640 / \lambda 4686$ ratio; Paper IV) show. If these differences, however, are not due to intrinsic reddening, then they represent a true difference in the energy balance of this NLS1 and that the X-ray emission is therefore consistent with the predictions of the slim disk models, indicating high accretion rates.

We estimated the luminosity in different energy bands using our parameterizations of the SED described above (SEDA and SEDB). Although with a $\sim 20 \%$ systematic error in the $0.3-$ $0.6 \mathrm{keV}$ band flux (C. Vignali 2003, private communication), the $X M M$ spectrum provides a way to extend the energy range below the $\sim 0.8 \mathrm{keV}$ limit of the $A S C A$ spectrum. Therefore, we also estimated the luminosity for the best-fit model to this spectrum. Table 5 reports these luminosities and indicates that more energy is emitted in the $10-100 \mathrm{eV}$ band than in any other decade, constituting roughly half of the emitted energy in the optical/X-ray ranges. This implies that the primary spectral component peaks in the extreme UV-soft X-ray band, and this is generally consistent with disk-corona models (Haardt \& Maraschi 1991). The integrated luminosity between
$10^{-5}$ and $10 \mathrm{keV}$ gives a lower limit on the bolometric luminosity of $L_{\mathrm{bol}} \gtrsim 10^{45} \mathrm{ergs} \mathrm{s}^{-1}$.

Growing evidence has been gathered that NLS1s are superEddington accretors. Collin \& Huré (2001) and Collin et al. (2002) studied accretion rates in a sample of AGNs by using the Kaspi et al. (2000) relationship and found not only that half of their sample accretes close to or above the Eddington rate but also that the largest Eddington ratios are found in NLS1s. Indeed, they showed that NLS1s are at the extreme of a welldefined sequence relating the Eddington ratio to the line widths. Furthermore, Wang \& Netzer (2003) show that a model of an extreme slim disk (which is responsible for the soft X-ray excess, or hump, seen in most NLS1s) and a hot corona (contributing to the hard X-ray emission) can also naturally explain the X-ray spectral variability characteristics observed in Ark 564, such as simultaneous variations of the soft hump and the hard X-ray without a significant time lag (Turner et al. 2001). Through a comparison of the X-ray variability of Ark 564 and the BLS1 NGC 3516, Pounds et al. (2001) estimate that the mass of the central black hole in Ark 564 is $\sim 10^{7} M_{\odot}$, implying an accretion rate in the range $\dot{m} \approx 0.2-1$. With a bolometric luminosity on the order of $10^{45} \mathrm{ergs} \mathrm{s}^{-1}$ and

TABLE 5

LUMINOSITIES

\begin{tabular}{ccccc}
\hline $\begin{array}{c}\text { Energy Range } \\
(\mathrm{keV}) \\
(1)\end{array}$ & $\begin{array}{c}L(\text { Observed }) \\
\left(\times 10^{44} \mathrm{ergs} \mathrm{s}^{-1}\right) \\
(2)\end{array}$ & $\begin{array}{c}L(\mathrm{SEDA})^{\mathrm{a}} \\
\left(\times 10^{44} \mathrm{ergs} \mathrm{s}^{-1}\right) \\
(3)\end{array}$ & $\begin{array}{c}L(\mathrm{SEDB})^{\mathrm{a}} \\
\left(\times 10^{44} \mathrm{ergs} \mathrm{s}^{-1}\right) \\
(4)\end{array}$ & $\begin{array}{c}L(\mathrm{XMM})^{\mathrm{a}} \\
\left(\times 10^{44} \mathrm{ergs} \mathrm{s}^{-1}\right) \\
(5)\end{array}$ \\
\hline $10^{-5}-10^{-4} \ldots \ldots \ldots$ & $\ldots$ & 2.1192 & 2.1192 & 2.1192 \\
$10^{-4}-10^{-3} \ldots \ldots .$. & $\ldots$ & 1.8108 & 1.8108 & 1.8108 \\
$10^{-3}-0.01 \ldots \ldots$. & $\ldots$ & 1.2464 & 1.2464 & 1.2464 \\
$0.01-0.1 \ldots \ldots \ldots$. & $\ldots$. & 3.5175 & 2.2589 & 4.1430 \\
$0.1-1 \ldots \ldots \ldots \ldots$. & 0.2201 & 1.6314 & 1.8080 & 2.7716 \\
$1-10 \ldots \ldots \ldots \ldots$. & 0.4086 & 0.4485 & 0.4485 & 0.5285 \\
$10^{-5}-10 \ldots \ldots \ldots$. & $\ldots$ & 10.7738 & 9.6918 & 12.6202 \\
\hline
\end{tabular}

Notes.-The SEDA luminosities have been calculated by using power-law parameterization of the SED with the spectral indices reported in Table 4. The SEDB luminosities refer to the more conservative parameterization described in $\S 3$ (Fig. 3). The $X M M$ luminosities make use of the $X M M$ spectrum in the $0.05-10 \mathrm{keV}$ band.

${ }^{a}$ Reddening-corrected, rest-frame luminosities. 
an Eddington luminosity $L_{\mathrm{Edd}} \approx 10^{45} \mathrm{ergs} \mathrm{s}^{-1}$, we also infer that $\dot{m} \approx 1$. Wang \& Netzer (2003) also provide a means of estimating the black hole mass (if the accretion is supercritical) that is independent of the accretion rate itself. For Ark 564, we obtain $M \approx 2 \times 10^{6} M_{\odot}$, which is within a factor of a few from the Collier et al. (2001) estimate.

Figure 8 compares the SED of Ark 564 with the mean SED for radio-quiet quasars (Elvis et al. 1994), the LZ SED (Laor et al. 1997; Zheng et al. 1997), the Seyfert 1.5 galaxies NGC 5548 (Kraemer et al. 1998) and NGC 4151 (Kraemer et al. 2000), and the NLS1 Ton S180 (Turner et al. 2002). There are significant differences in the intrinsic shape of the SED across the AGN population (see also Turner et al. 2002), the most evident being the energy of the peak and the presence (or lack) of the big blue bump (BBB), the signature of the emission from the accretion disk.

The radial dependence of the temperature for an optically thick, geometrically thin accretion disk (Shakura \& Sunyaev $1973)$ is $T(R) \sim 6.3 \times 10^{5}(\dot{m})^{1 / 4} M_{8}^{-1 / 4}\left(R / R_{\mathrm{S}}\right)^{-3 / 4} \mathrm{~K}$ (Peterson et al. 2000), where $M_{8}$ is the mass in units of $10^{8} M_{\odot}, R$ is the radius, and $R_{\mathrm{S}}$ is the Schwarzschild radius. For Ark 564, using $\dot{m} \approx 1$, as we derived above, and $M \approx 8 \times 10^{6} M_{\odot}$ (Paper II), the peak temperature is $\sim 125 \mathrm{eV}$; this is within a factor of 3 from the peak of our less conservative parameterization, SEDA (defined by extrapolating the optical powerlaw continuum to meet the extrapolation of the X-ray power law), which peaks at $50 \mathrm{eV}$. The true SED probably peaks somewhere between these two values. For comparison, the NLS1s RE J1034+396 (Puchnarewicz et al. 2001) and Ton S180 (Turner et al. 2002) peak at $\approx 250$ and $\lesssim 100 \mathrm{eV}$, respectively. Therefore, even among the NLS1s, differences in the shape of the SED are observed. However, in none of these NLS1s there is an indication of the presence of optical/UV $\mathrm{BBB}$, and a strong soft X-ray excess is seen, instead. In this

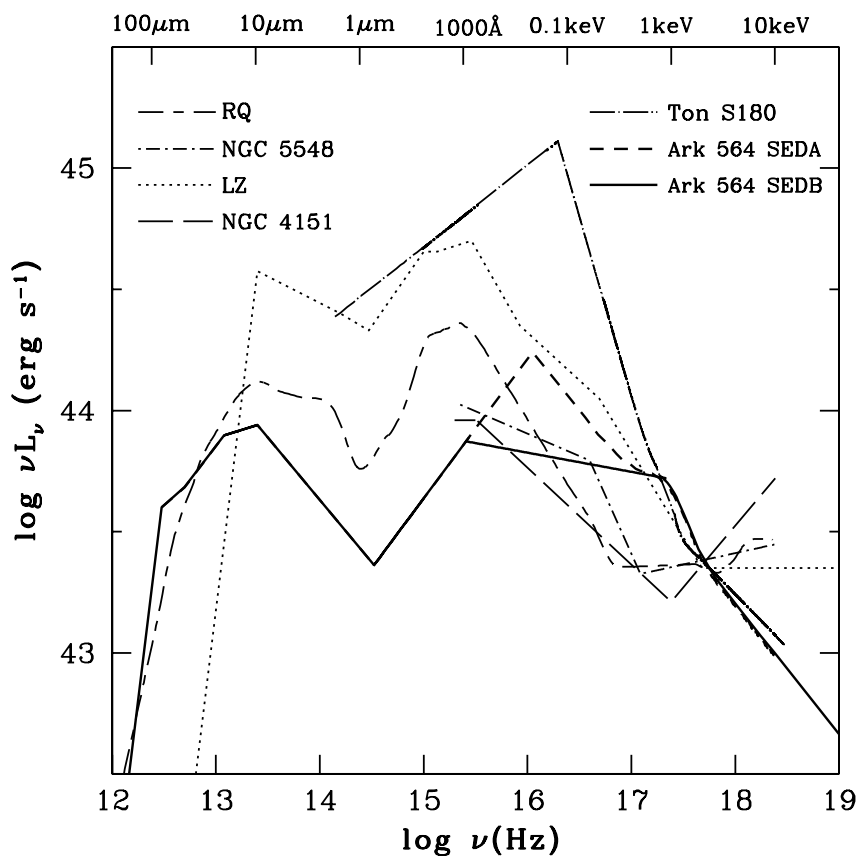

FIG. 8.-Comparison of the SEDs of Ark 564 with the mean SED for radioquiet quasars (RQ; Elvis et al. 1994) and LZ (Laor et al. 1997; Zheng et al. 1997), one Seyfert 1 galaxy (NGC 4151; Kraemer et al. 2000), one Seyfert 1.5 galaxy (NGC 5548; Kraemer et al. 1998), and the NLS1 Ton S180 (Turner et al. 2002). [See the electronic edition of the Journal for a color version of this figure.]

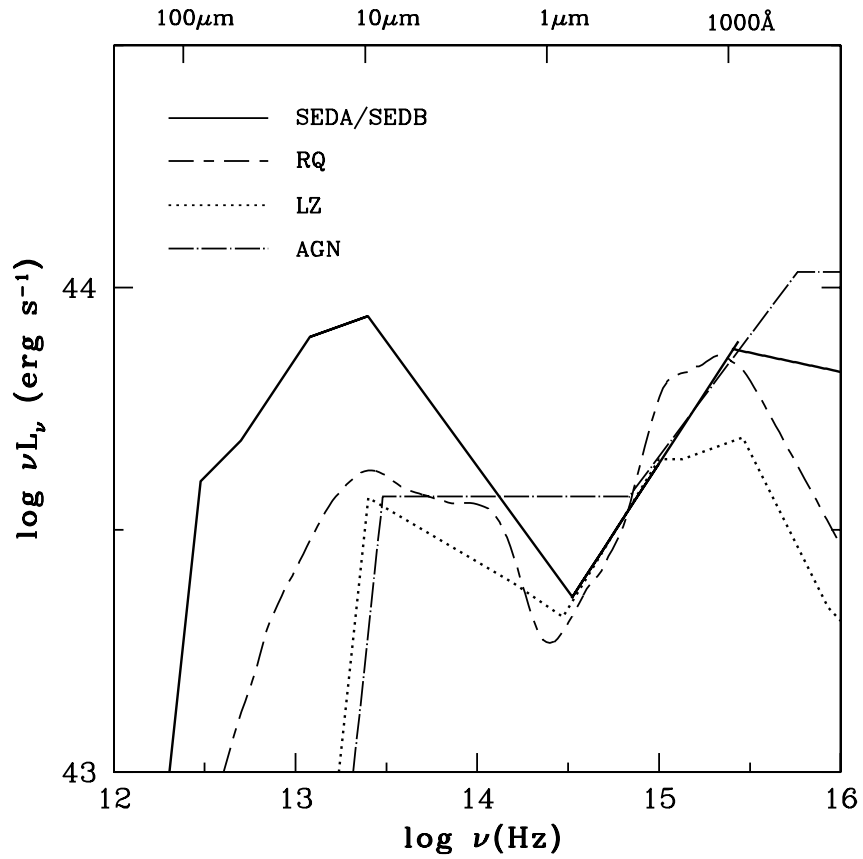

FIG. 9.-Comparison of the Ark 564 SED with the mean SED for radioquiet quasars (RQ; Elvis et al. 1994), LZ (Laor et al. 1997; Zheng et al. 1997), and the table AGN model in CLOUDY (AGN). The SEDs are normalized so that they match in the optical/UV part.

light, Ark 564 is also consistent with the paradigm that the accretion disk is so hot in NLS1s that the BBB is shifted in the EUV-soft X-rays.

We also note that Ark 564 is rather FIR-bright (with respect to the optical) compared with the sample of radio-quiet quasars and the LZ sample, as can be seen in Figure 9, where the SEDs have been normalized to match their optical/UV slope (as opposed to the $2 \mathrm{keV}$ flux in Fig. 8). Indeed, if we use the definition of the IR flux as a function of the IRAS fluxes given by Sanders \& Mirabel (1996), ${ }^{24}$ we obtain that $L(8-1000 \mu \mathrm{m}) \approx$ $10^{11} L_{\odot}$, which makes Ark 564 a luminous IR galaxy. The shape of the IR/optical SED of Ark 564 also resembles the shapes observed in the IRAS Bright Galaxy Survey (see Fig. 2 in Sanders \& Mirabel 1996 for intermediate values of $f_{60}$ ). Using SEDA, we estimate that the FIR luminosity is $L_{\mathrm{FIR}} \approx$ $1.3 \times 10^{44}$ ergs $\mathrm{s}^{-1}$, i.e., $\sim 10 \%$ of the total luminosity and $\sim 20 \%$ of the combined optical/UV/X-ray luminosity. Crenshaw et al. (2002) noted that the associated warm UV absorber is lukewarm and dusty. The IR emission observed in this object could then be thermal emission from the dust grains embedded in the absorber as they are heated by the strong UV/EUV continuum. Given the IR brightness in this object and in many NLS1s (Moran, Halpern, \& Helfand 1996), it is not unlikely that a contribution might be coming from the host galaxy, in the form of a nuclear starburst (Mathur 2000). Crenshaw et al. (2002), however, did not detect any extended emission in the two-dimensional STIS spectral images that could be due to a nuclear starburst. We compared the FUSE spectrum, which was taken through a much larger aperture and hence is more likely to show stellar absorption features, with a FUSE "template" spectrum of the

\footnotetext{
${ }^{24} F(8-1000 \mu \mathrm{m})=1.8 \times 10^{-14}\left(13.48 f_{12}+5.16 f_{25}+2.58 f_{60}+f_{100}\right) \mathrm{W} \mathrm{m}^{-2}$, where $f_{12}, f_{25}, f_{60}$, and $f_{100}$ are the IRAS flux densities in Jy at $12,25,60$, and $100 \mu \mathrm{m}$.
} 
starburst galaxy NGC 7496; using the constraints from the Ly $\gamma-\mathrm{C}$ III] $\lambda 977$ profiles, we obtained a rough upper limit of the starburst contribution at $1000 \AA$ of $50 \%$. A further constraint on the IR starburst contribution would probably come from detection and measurements of the $3.3-3.4 \mu \mathrm{m}$ polycyclic aromatic hydrocarbon emission features, which have been found in starburst galaxies, luminous IR galaxies, and obscured AGNs (Moorwood 1986; Imanishi 2002) and which have been successfully detected in the NLS1 NGC 4051 (RodríguezArdila \& Viegas 2003).

Using our SEDA and SEDB as inputs to CLOUDY, we predicted the intensity of the strongest lines in the FUV/UV spectrum and compared them with the observed values in order to constrain the physical parameters of the line-emitting gas, namely, the density and ionization parameter. Figures 6 and 7 show that SEDA and SEDB, because of their strong EUV to soft X-ray flux, for a given observed line ratio predict values of ionization parameter that are lower than those with standard AGN continuum. From C III] $\lambda 1909$, C III $\lambda 2977$, and $\mathrm{N} \vee \lambda 1240$, we infer that $\log n \approx 11$. Two classes of solutions for $U$ are consistent with this density value, one with low $U$ values $(\log U \approx-1.5)$ and one with high $U$ values $(\log U \approx 0)$. We discarded the low- $U$ class $(\S 4.3)$ on the basis that the predicted widths of the lines, derived from the velocity dispersion $V=\left(G M / R_{\mathrm{BLR}} f\right)^{0.5}$, of $740-880 \mathrm{~km} \mathrm{~s}^{-1}$ are too small with respect to the observed ones $(\approx 2000 \mathrm{~km}$ $\mathrm{s}^{-1}$; Paper II). As expected, we find that the BLR is stratified around $\log U \approx 0$, with higher ionization lines originating from regions with higher $U$.

Column (6) of Table 3 shows that FWHM $(\operatorname{Ly} \alpha)=2114 \mathrm{~km}$ $\mathrm{s}^{-1}, \operatorname{FWHM}(\mathrm{N} \mathrm{v})=2809 \mathrm{~km} \mathrm{~s}^{-1}$, and $\operatorname{FWHM}(\mathrm{C} \mathrm{IV})=$ $1934 \mathrm{~km} \mathrm{~s}^{-1}$, which indicate that the radii of the $\operatorname{Ly} \alpha, \mathrm{N} \mathrm{v}$, and $\mathrm{C}$ IV broad-line-emitting regions are $R_{\mathrm{BLR}}^{\mathrm{Ly} \alpha} \approx 4.3$ lt-days, $R_{\mathrm{BLR}}^{\mathrm{NV}} \approx 2.5$ lt-days, and $R_{\mathrm{BLR}}^{\mathrm{C} \text { IV }} \approx 5.2$ 1t-days. Using the findings of previous monitoring programs on Seyfert 1 galaxies (Netzer \& Peterson 1997), we can estimate the size of the $\mathrm{H} \beta$ emitting region from $R_{\mathrm{BLR}}^{\mathrm{Ly} \alpha} \approx 0.5 R_{\mathrm{BLR}}^{\mathrm{H} \beta}$ and $R_{\mathrm{BLR}}^{\mathrm{NV}} \approx 0.2 R_{\mathrm{BLR}}^{\mathrm{H} \beta}$. For NGC 5548, furthermore, $R_{\mathrm{BLR}}^{\mathrm{C} \text { IV }} \approx 0.5 R_{\mathrm{BLR}}^{\mathrm{H} \beta}$ (Peterson 1993). We can conclude that $R_{\mathrm{BLR}}^{\mathrm{H} \beta} \approx 10 \pm 2$ lt-days, which is consistent with the $R_{\mathrm{BLR}}^{\mathrm{HB}}$-luminosity relationships of Kaspi et al. (2000) and Peterson et al. (2000) when we assume a luminosity $\lambda L_{\lambda}(5100 \AA) \approx 3.2 \times 10^{43} \mathrm{ergs} \mathrm{s}^{-1}$ (Table 2 ). This indicates that the BLR radius of this NLS1 is consistent with the distribution of BLR radius in BLS1s and that the narrowness of the emission lines is not due to the BLR being relatively farther away from the central mass than in BLS1s of comparable luminosity.

Table 3 shows that some interesting differences in line ratios can be found with respect to the Seyfert 1.5 NGC 5548. Indeed, $\mathrm{N} v \lambda 1240$ is stronger in Ark 564 by a factor of $\sim 2.3$, while $\left.\mathrm{C}_{\text {IV }} \lambda 1550, \mathrm{C}_{\mathrm{III}}\right] \lambda 1909$, and $\mathrm{Mg}$ II $\lambda 2800$ are weaker in Ark 564 by a factor of $\sim 4.4,2.5$, and 2.8 , respectively. While all line ratios in Ark 564 are statistically consistent with the ones measured for a mean QSO (given the large uncertainties on our measurements), carbon lines are at the lower end and nitrogen at the upper end of the QSO distribution, confirming this trend for weak carbon and strong nitrogen in Ark 564. Furthermore, C III] 2977 would indicate that $-3.13<\log U<-0.05$ (Fig. 6), and for this range, the observed $\mathrm{N}$ v/C IV ratio is larger than the model predictions by a factor of $\sim 8$. This may imply supersolar metallicity in this NLS1 as suggested by Mathur (2000) and is consistent with the finding of Shemmer \& Netzer (2002) that NLS1s have higher metallicities than broad-line AGNs for a given luminosity.
An interesting question is how sensitive the emission lines are to the true shape of the ionizing continuum. Given the difficulty related to deblending the BEL and NEL components of the lines and consequent large errors on the observed line ratios, the present emission-line data do not allow us to discriminate between SEDA and SEDB. These SEDs differ in the range $1000 \AA$ to $750 \mathrm{eV}$ (corresponding to the gap in the data between the FUSE and ASCA spectra), with the maximum difference at around $50 \mathrm{eV}$. This difference should show the most for the high-ionization lines of $\mathrm{C}$ IV, $\mathrm{N} \mathrm{v}$, and $\mathrm{O}$ VI. However, the predicted strength of their emission lines is very similar for the two SEDs, for a wide range of ionization parameters of interest (Figs. 6 and 7). Perhaps this is the reason why the emission-line spectra of most AGNs look so very similar over a wide range of luminosities. This demonstrates how unsuitable emission lines are as diagnostics for the underlying SEDs.

The absorption lines, on the other hand, are sensitive to the input SED (Mathur et al. 1994). Column densities of different ions can be inferred from the fractional abundances $f_{\text {ion }}$, the total column density $N_{\mathrm{H}}$, and the assumed abundances $N_{\mathrm{X}}$, $N_{\text {ion }}=N_{\mathrm{H}} N_{\mathrm{X}} N_{\text {ion }}$, through photoionization calculations (assuming an SED). Figure 10 (top) shows the fractional column densities for the same ion calculated with the different SEDs $\left[N_{\mathrm{C} \text { IV }}(\mathrm{SEDA}) / N_{\mathrm{C} \text { Iv }}(\mathrm{SEDB}), N_{\mathrm{N} \mathrm{v}}(\mathrm{SEDA}) / N_{\mathrm{N} \mathrm{v}}(\mathrm{SEDB})\right.$, $N_{\mathrm{O} V \mathrm{VI}}(\mathrm{SEDA}) / N_{\mathrm{O} \text { VI }}(\mathrm{SEDB})$, and $N_{\mathrm{O} \text { VII }}(\mathrm{SEDA}) / N_{\mathrm{O} \text { VII }}$ (SEDB)] and illustrates how the predicted column densities of highionization lines differ, with $\mathrm{O}$ vI showing the largest variations, with over 2 orders of magnitude difference. Figure 10 (bottom) compares the column density for $\mathrm{O}$ VII and $\mathrm{C}$ IV calculated with the same SED and solar abundances $\left[N_{\mathrm{O} \text { VII }}\right.$ (SEDA)/ $N_{\mathrm{C} \text { IV }}(\mathrm{SEDA})$ and $\left.N_{\mathrm{O} \text { VII }}(\mathrm{SEDB}) / N_{\mathrm{C} \mathrm{IV}}(\mathrm{SEDB})\right]$, which turn out to be good diagnostics even at lower ionization parameters. Thus, in principle, absorption-line studies offer a far more powerful tool to determine the ionizing continuum of AGNs, compared with the emission lines. This also underscores the

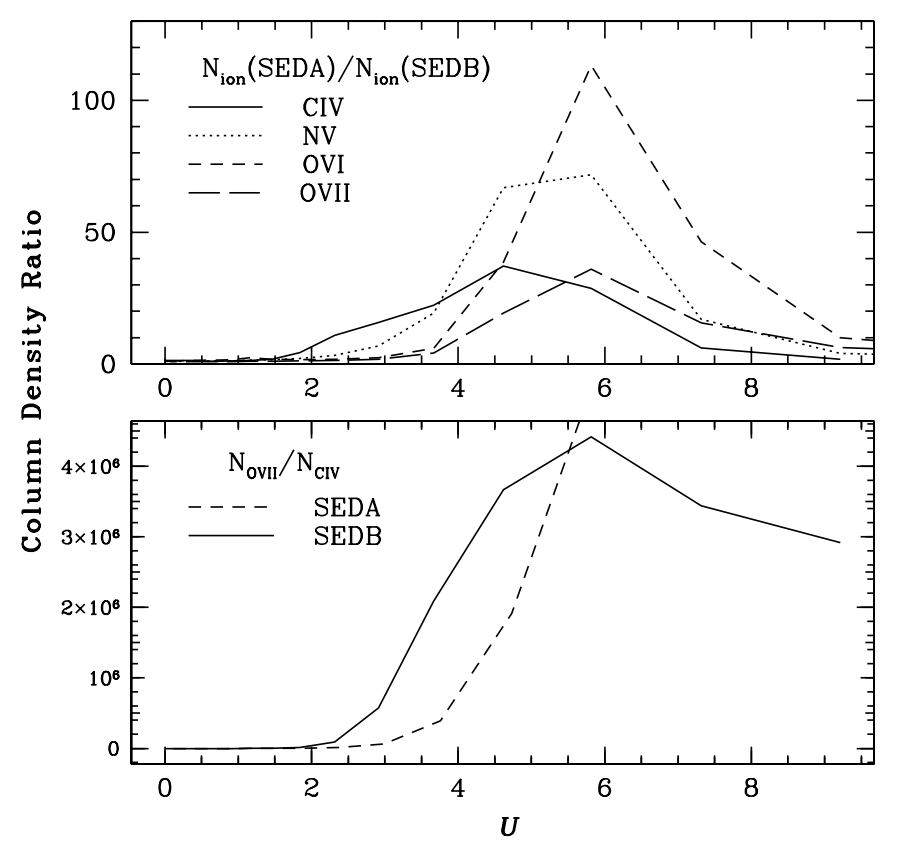

FIG. 10.-Top: Ratio of the column densities relative to SEDA and SEDB as a function of $U$ for $\mathrm{C}$ rv, $\mathrm{N}$ v, $\mathrm{O}$ vI, and $\mathrm{O}$ vII. Bottom: Ratio of the column densities of $\mathrm{O}$ VII to $\mathrm{C}$ IV as a function of $U$ for solar abundances, for SEDA and SEDB. 
power of multiwavelength observations, as the maximum discriminator comes from comparing the lower and higher ionization lines.

\section{SUMMARY}

We presented the intrinsic spectral energy distribution of Ark 564, constructed with quasi-simultaneous data obtained during 2000 and 2001. We compared this SED with that of Ton S180 and with those obtained for broad-line Seyfert 1 galaxies to infer how the relative accretion rates vary among the Seyfert 1 population. The peak of the SED is not well constrained; however, in our parameterization most of the energy of this object is emitted in the $10-100 \mathrm{eV}$ regime and constitutes roughly half of the emitted energy in the optical/X-ray ranges. This is consistent with a primary spectral component peaking in the soft X-ray band and therefore with the predictions of the slim disk models, hence high accretion rates. Indeed, we estimate that $\dot{m} \approx 1$.

We constrained the mean physical conditions in the BELR of this AGN by examining the emission lines observed in its spectrum and deduced the physical properties of the lineemitting gas through photoionization modeling. We concluded that the line-emitting gas is characterized by $\log n \approx 11$ and $\log U \approx 0$ and is stratified around $\log U \approx 0$. Our estimate of the radius of the $\mathrm{H} \beta$-emitting region $R_{\mathrm{BLR}}^{\mathrm{H} \beta} \approx 10 \pm 2$ lt-days is consistent with the $R_{\mathrm{BLR}}^{\mathrm{H} \beta}$-luminosity relationships of Kaspi et al. (2000) and Peterson et al. (2000). This indicates that the narrowness of the emission lines is not due to the BLR being relatively farther away from the central mass than in BLS1s of comparable luminosity. We also find evidence for supersolar metallicity in this NLS1, based on the low C Iv $/ \mathrm{N} \mathrm{v}$ observed line ratio. While the emission lines turn out to be unsuitable as diagnostics for the underlying SEDs, we showed that absorption-line studies offer a far more powerful tool to determine the ionizing continuum of AGNs, especially if one compares the lower and higher ionization lines. This underlines the power of multiwavelength observations.

We thank C. Vignali for providing us with the $X M M$ spectrum ahead of publication and the anonymous referee for suggestions that improved the paper. P. R. and S. M. acknowledge support through NASA grant NAG5-10320 (FUSE) and the Italian MIUR. T. J. T. and W. N. B. acknowledge support through NASA LTSA grants NAG5-7385 and NAG5-13035, respectively. We also acknowledge support from HST-GO-08265.01-A from the Space Science Telescope Institute, which is operated by the Association of Universities for Research in Astronomy, Inc., under NASA contract NSS5226555. This research has made use of the NASA/IPAC Extragalactic Database (NED), which is operated by the Jet Propulsion Laboratory, California Institute of Technology, under contract with the National Aeronautics and Space Administration.
Baldwin, J., Ferland, G., Korista, K., \& Verner, D. 1995, ApJ, 455, L119

Boller, Th., Brandt, W. N., \& Fink, H. 1996, A\&A, 305, 53

Brandt, W. N., Fabian, A. C., Nandra, K., Reynolds, C. S., \& Brinkmann, W. 1994, MNRAS, 271, 958

Brandt, W. N., Mathur, S., \& Elvis, M. 1997, MNRAS, 285, L25

Brotherton, M. S., Green, R. F., Kriss, G. A., Oegerle, W., Kaiser, M. E., Zheng, W., \& Hutchings, J. B. 2002, ApJ, 565, 800

Cardelli, J. A., Clayton, G. C., \& Mathis, J. S. 1989, ApJ, 345, 245

Cheng, F. H., Gaskell, C. M., \& Koratkar, A. P. 1991, ApJ, 370, 487

Collier, S. J., et al. 2001, ApJ, 561, 146 (Paper II)

Collin, S., Boisson, C., Mouchet, M., Dumont, A.-M., Coupé, S., Porquet, D., \& Rokaki, E. 2002, A\&A, 388, 771

Collin, S., \& Huré, J.-M. 2001, A\&A, 372, 50

Comastri, A., et al. 2001, A\&A, 365, 400

Constantin, A., \& Shields, J. C. 2003, PASP, 115, 592

Contini, M., Rodríguez-Ardila, A., \& Viegas, S. M. 2003, A\&A, 408, 101

Crenshaw, D. M., Kraemer, S. B., Boggess, A., Maran, S. P., Mushotzky, R. F., $\&$ Wu, C. 1999, ApJ, 516, 750

Crenshaw, D. M., et al. 2002, ApJ, 566, 187 (Paper IV)

de Vaucouleurs, G., de Vaucouleurs, A., Corwin, H. G., Jr., Buta, R. J., Paturel, G., \& Fouqué, P. 1991, S\&T, 82, 621

Dickey, J. M., \& Lockman, F. M. 1990, ARA\&A, 28, 215

Edelson, R., et al. 2002, ApJ, 568, 610

Elvis, M., et al. 1994, ApJS, 95, 1

Ferland, G. J. 1996, Hazy, a Brief Introduction to CLOUDY 94.00 (Univ. Kentucky Dept. Phys. Astron. Int. Rep.)

Goodrich, R. W. 1989, ApJ, 342, 224

Grupe, D., Beuermann, K., Thomas, H.-C., Mannheim, K., \& Fink, H. H. 1998, A\&A, 330, 25

Haardt, F., \& Maraschi, L. 1991, ApJ, 380, L51

Hutchings, J. B., \& Giasson, J. 2001, PASP, 113, 1205

Imanishi, M. 2002, ApJ, 569, 44

Kaspi, S., Smith, P. S., Netzer, H., Maoz, D., Jannuzi, B. T., \& Giveon, U. 2000, ApJ, 533, 631

Korista, K., et al. 1995, ApJS, 97, 285

Korista, K. T., \& Goad, M. R. 2000, ApJ, 536, 284

Kraemer, S. B., Crenshaw, D. M., Filippenko, A. V., \& Peterson, B. M. 1998, ApJ, 499, 719

Kraemer, S. B., Crenshaw, D. M., Hutchings, J. B., Gull, T. R., Kaiser, M. E., Nelson, C. H., \& Weistrop, D. 2000, ApJ, 531, 278

\section{EFERENCES}

Laor, A., Fiore, F., Elvis, M., Wilkes, B. J., \& McDowell, J. C. 1997, ApJ, 477, 93

Lawrence, A., Elvis, M., Wilkes, B. J., McHardy, I., \& Brandt, N. 1997, MNRAS, 285, 879

Leighly, K. M. 1999a, ApJS, 125, 297

1999b, ApJS, 125, 317

Lindler, D. 1998, CALSTIS Reference Guide (CALSTIS Version 5.1) (Greenbelt: NASA)

Maraschi, L., \& Haardt, F. 1997, in IAU Colloq. 163, Accretion Phenomena and Related Outflows, ed. D. Wickramasinghe, L. Ferrario, \& G. Bicknell (San Francisco: ASP), 101

Mathews, W. G., \& Ferland, G. J. 1987, ApJ, 323, 456

Mathur, S. 2000, MNRAS, 314, L17

Mathur, S., Wilkes, B., Elvis, M., \& Fiore, F. 1994, ApJ, 434, 493

Matsumoto, C., Leighly, K. M., \& Marshall, H. L. 2002, in X-Ray Spectroscopy of AGN with Chandra and XMM-Newton, ed. T. Boller (Garching: MPE), 263

Moorwood, A. F. M. 1986, A\&A, 166, 4

Moran, E. C., Halpern, J. P., \& Helfand, D. J. 1996, ApJS, 106, 341

Moshir, M., et al. 1990, IRAS Faint Source Catalog, Version 2.0 (Pasadena: Infrared Processing and Analysis Center)

Nagao, T., Murayama, T., \& Taniguchi, Y. 2001, ApJ, 546, 744

Nandra, K., George, I. M., Mushotzky, R. F., Turner, T. J., \& Yaqoob, T. 1997a, ApJ, 476, 70 1997b, ApJ, 477, 602

Netzer, H., \& Peterson, B. M. 1997, in Astronomical Time Series, ed. D. Maoz \& A. Sternberg (Dordrecht: Kluwer), 85

Osterbrock, D. E., \& Pogge, R. W. 1985, ApJ, 297, 166

Peterson, B. M. 1993, PASP, 105, 247

Peterson, B. M., \& Wandel, A. 1999, ApJ, 521, L95

Peterson, B. M., et al. 2000, ApJ, 542, 161

Pounds, K. A., Done, C., \& Osborne, J. P. 1995, MNRAS, 277, L5

Pounds, K. A., Edelson, R., Markowicz, A., \& Vaughan, S. 2001, ApJ, 550, L15

Puchnarewicz, E. M., Mason, K. O., Siemiginowska, A., Fruscione, A., Comastri, A., Fiore, F., \& Cagnoni, I. 2001, ApJ, 550, 644

Puchnarewicz, E. M., et al. 1996, MNRAS, 281, 1243

Rodríguez-Ardila, A., Binette, L., Pastoriza, M. G., \& Donzelli, C. J. 2000, ApJ, 538, 581

Rodríguez-Ardila, A., \& Viegas, S. M. 2003, MNRAS, 340, L33 
Rodríguez-Ardila, A., Viegas, S. M., Pastoriza, M. G., \& Prato, L. 2002a, ApJ, 565,140

2002b, ApJ, 579, 214

Romano, P., Mathur, S., Pogge, R. W., Peterson, B. M., \& Kuraszkiewicz, J. 2002, ApJ, 578, 64 (Paper V)

Ross, R., Fabian, A. C., \& Mineshige, S. 1992, MNRAS, 258, 189

Sanders, D. B., \& Mirabel, I. F. 1996, ARA\&A, 34, 749

Sasseen, T. P., Hurwitz, M., Dixon, W. V., \& Airieau, S. 2002, ApJ, 566, 267

Shakura, N. I., \& Sunyaev, R. A. 1973, A\&A, 24, 337

Shemmer, O., et al. 2001, ApJ, 561, 162 (Paper III)

Shemmer, O., \& Netzer, H. 2002, ApJ, 567, L19

Turner, T. J., George, I. M., \& Nandra, K. 1998, ApJ, 508, 648

Turner, T. J., Romano, P., George, I. M., Edelson, R., Collier, S. J., Mathur, S., \& Peterson, B. M. 2001, ApJ, 561, 131 (Paper I)

Turner, T. J., et al. 1999a, ApJ, 510, 178
Turner, T. J., et al. 1999b, in Proc. 19th Texas Symp. on Relativistic Astrophysics and Cosmology, ed. J. Paul, T. Montmerle, \& E. Aubourg (Saclay: CEA), E441 2002, ApJ, 568, 120

Vaughan, S., Reeves, J., Warwick, R., \& Edelson, R. 1999, MNRAS, 309, 113

Véron-Cetty, M.-P., Véron, P., \& Gonçalves, A. C. 2001, A\&A, 372, 730

Vignali, C., Brandt, W. N., Boller, Th., Fabian, A. C., \& Vaughan, S. 2004, MNRAS, in press (astro-ph/0310278)

Walter, R., \& Fink, H. H. 1993, A\&A, 274, 105

Wang, J.-M., \& Netzer, H. 2003, A\&A, 398, 927

Yaqoob, T., et al. 2000, ASCA GOF Calibration Memo (ASCA-CAL-00-06-01, ver. 1.0)

Zamorani, G., et al. 1981, ApJ, 245, 357

Zheng, W., Kriss, G. A., Telfer, R. C., Grimes, J. P., \& Davidsen, A. F. 1997, ApJ, 475, 469 